AFRL-SN-RS-TR-2005-224

Final Technical Report

June 2005

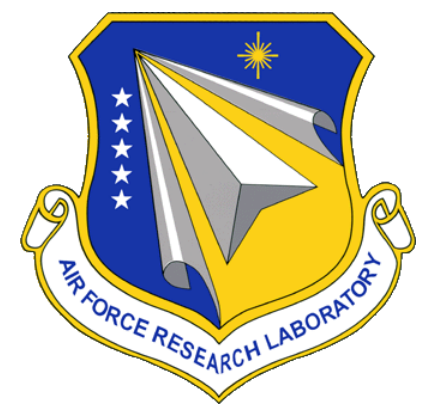

\title{
ALL SOLID-STATE PHOTOMIXING THZ TRANSMITTER
}

University of California, Los Angeles

APPROVED FOR PUBLIC RELEASE; DISTRIBUTION UNLIMITED.

\author{
AIR FORCE RESEARCH LABORATORY \\ SENSORS DIRECTORATE \\ ROME RESEARCH SITE \\ ROME, NEW YORK
}




\title{
STINFO FINAL REPORT
}

This report has been reviewed by the Air Force Research Laboratory, Information Directorate, Public Affairs Office (IFOIPA) and is releasable to the National Technical Information Service (NTIS). At NTIS it will be releasable to the general public, including foreign nations.

AFRL-SN-RS-TR-2005-224 has been reviewed and is approved for publication

\author{
APPROVED: /S/ \\ JAMES NICHTER \\ Project Engineer
}

FOR THE DIRECTOR:

/S/

RICHARD G. SHAUGHNESSY

Chief, Rome Operations Office

Sensors Directorate 


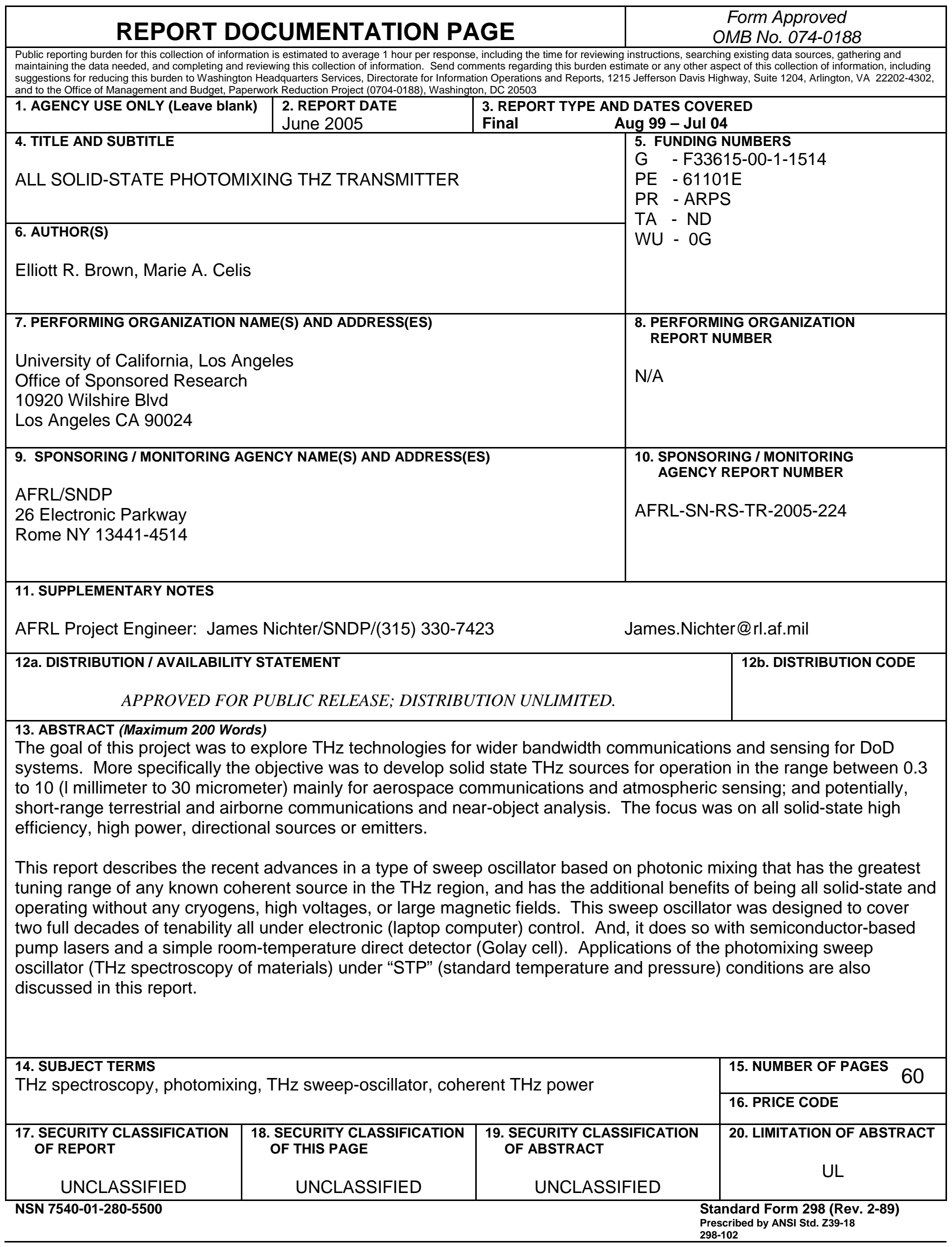




\section{Table of Contents}

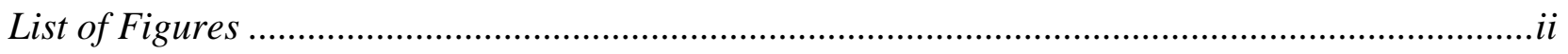

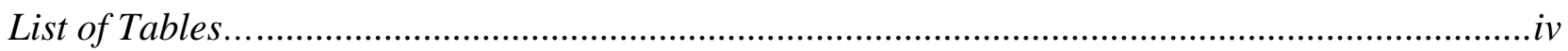

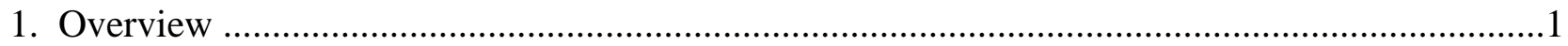

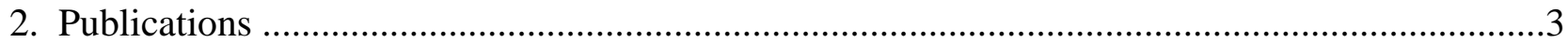

2.1. Manuscripts submitted, but not published ...................................................................3

2.2. Papers published in peer-reviewed journals ..................................................................

2. 3. Recent Book Chapters..............................................................................................4

2.4. Papers presented at meetings, but not published in conference proceedings......................4

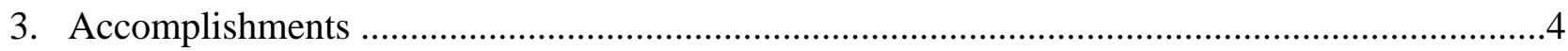

3.1. Background on Coherent Sources .............................................................................4

3.2. Coherent $\mathrm{THz}$ Power by Photomixing..........................................................................8

3.2.1. Photomixing and photoconductor measurements on ErAs/GaAs at $780 \mu$ m..............12

3.2.2. Photomixing and photoconductor measurements on ErAs/InGaAs at $1.55 \mu \mathrm{m}$............21

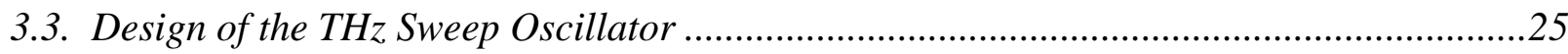

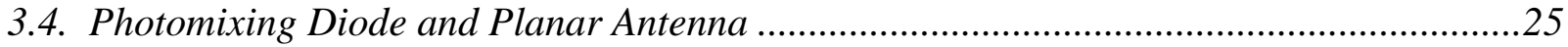

3.5. Optical coupling to free space and to the sample ........................................................27

3.6. Receivers: Golay Cell and hot-electron Bolometer.....................................................31

3.7. Pump Lasers and THz Frequency Monitoring..........................................................37

3.8. Control and Signal Processing ................................................................................40

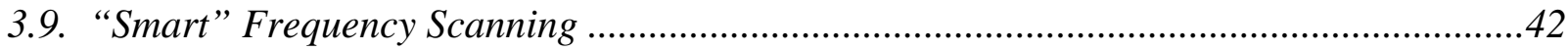

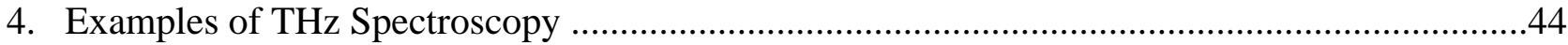

4.1. Fast-scan, broadband mode ..................................................................................44

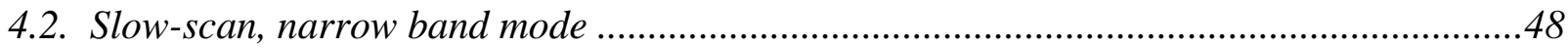

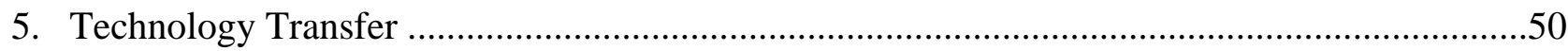

6. Scientific personnel" supported by this project and honors/awards/degrees received ...............51

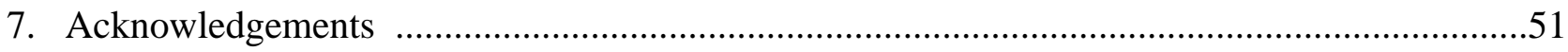

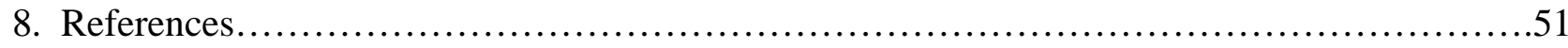




\section{List of Figures}

Fig. 1 Approximate average output power of various $\mathrm{THz}$ sources in use today..................6

Fig. 2 Schematic diagram of interdigitated photomixer coupled to a strip line antenna, and simplified equivalent circuit in terms of a THz current source coupled to an antenna of radiation resistance $\mathrm{RA}$.

Fig. 3 Cross-sectional view of interdigitated-electrode photomixer illuminated from the air side by two frequency-offset laser beams.

Fig. 4 Microphotograph of interdigitated electrode photomixer coupled to a two-turn selfcomplementary rectangular-spiral antenna....

Fig. 5 Photomixing frequency curve for pump lasers centered at 780 and $1550 \mathrm{~nm} . . \ldots \ldots \ldots \ldots \ldots 11$

Fig. 6 Cross section of photomixer device...............................................

Fig. 7 Experimental setup used to characterize ErAs:GaAs photomixers......................14

Fig. 8 Current vs. Voltage in ErAs:GaAs photomixer. The dark current was measured without optical pumping (dark) and the photocurrent with a $30 \mathrm{~mW}$ optical power at $\Gamma$ (photocurrent).

Fig. 9 Current vs. Voltage extracted from a curve tracer connected to ErAs:GaAs photomixer illuminated with 20 and $40 \mathrm{~mW}$ laser.

Fig. 10 Output power of photomixer vs. Laser pump power under constant bias conditions $(\mathrm{V}=24,27$ and 30) and offset frequency $\Delta \mathrm{f}=88$.

Fig. 11 Output power from a photomixer driven dipole-spiral antenna detected with a Golay cell vs. frequency offset of incident lasers. .21

Fig. 12 Material Structure: ErAs:GaAs..............................................22

Fig. 13 Plot of Current vs. Voltage in ErAs/InGaAs Photoconductor...........................23

Fig. 14 Responsivity as a Function of Vbias.........................................23

Fig. 15 Power vs. Offset Frequency.....................................................24

Fig. 16(a) Cross sectional view of photomixer chip coupled to silicon hyperhemisphere. 16(b) Gaussian-beam propagation diagram showing the 1/e intensity locus and the longitudinal position of all the optical elements between the photomixer and the bolometer power meter.

Fig. 17 Block diagram of the key components of the photomixing spectrometer. 
Fig. 18 Cryogenic detector apparatus used in consisting of an magnetically-enhanced INSb hot-electron bolometer cooled by a helium closed-cycle refrigerator and coupled to free space with a brass conical feedhorn

Fig. 19 Schematic diagram of low-noise amplifier used with hot-electron bolometer that provides an input noise voltage of $\sim 1 \mathrm{nV} / \mathrm{Hz}^{1 / 2}$

Fig. 20 Optical power spectrum of tunable and fixed-frequency MOPA lasers taken with confocal Fabry-Perot spectrometer.

Fig. 21 Power spectrum of tunable and fixed-frequency MOPA lasers taken with fiber-optically-coupled optical spectrum analyzer.

Fig. 22 Photomixer output power vs offset frequency as measured by the Golay cell and recorded by the lock-in amplifier. The scan from $100 \mathrm{GHz}$ to $1 \mathrm{THz}$ is made with an integration time $\tau$ of $300 \mathrm{~ms}$, and the scan above $1 \mathrm{THz}$ is made with a $\tau$ of $1 \mathrm{~s}$, yielding an approximately constant signal-to-noise ratio (CSNR) across the entire scan....43

Fig. 23 Example of the use of photomixer spectroscopy on common organic and biomaterials. All scans were made in fast-scan broadband (i.e., low resolution) mode

Fig. 24 The attenuation coefficient of potato starch vs frequency derived from its fast-scan broadband transmission spectrum. Also shown is a quadratic frequency dependence fit.

Fig. 25 Example of a 1-THz scan taken with photomixing spectrometer in slow-scan, narrow-band mode. The medium is the atmosphere in our laboratory over a path length of $15.5 \mathrm{~cm}$.

Fig. 26 The same scan as Fig. 24 with zoom-in on two of the strong water-vapor absorption signatures 


\section{List of Tables}

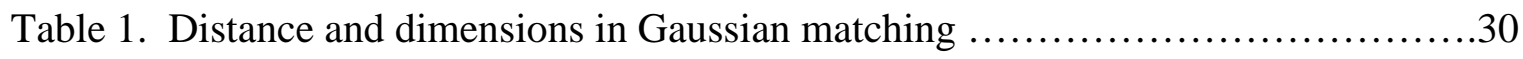

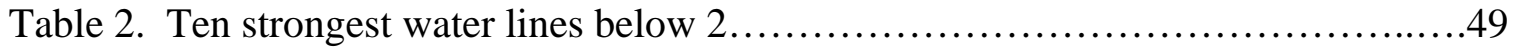




\section{Overview}

The electromagnetic spectrum from approximately 300 Gigahertz (GHz) to 10 Terahertz (THz) has been scientifically rich in the realms of molecular chemistry and astrophysics with substantial technological importance. This spectral region represents a significant part of the gap separating electronics, oriented towards transport, from photonics, oriented towards quantum transitions. Developing $\mathrm{THz}$ technology will bring benefits to advanced DOD systems. But it has not been broadly utilized to make systems for commercial or military applications largely because of the difficulties in generating and propagating coherent $\mathrm{THz}$ radiation. As in the lower RF frequency bands, one of the enabling instruments is a coherent source that can continuously tune over a very broad frequency range, commonly known as a sweep oscillator.

The region of the electromagnetic spectrum offers various technological opportunities and challenges. It offers the potential for a number of applications including space-based and short-range terrestrial or near earth communications, atmospheric sensing, collision avoidance for aircraft and ground vehicles, and near object observation and spectroscopy. There is a continuing need for ultrahigh bandwidth communications and sensing that requires electronics that operate at $\mathrm{THz}$ frequencies. Given that advanced microwave satellite communications already operate near $60 \mathrm{GHz}$, the instantaneous bandwidth required to fully monitor the battle space will certainly exceed $300 \mathrm{GHz}$ in the 21st century. All communication bottlenecks must be removed so that surveillance systems can relay their wideband measurements to other locations for real-time analysis.

THz technology will dramatically improve many aspects of covert communication and battlefield imaging. Increased atmospheric absorption observed over this range of frequencies give rise to applications in secure ultra-high bandwidth communications. A single THz satellite communication link will support broadband data transfer rates far beyond ( $>20 \mathrm{X})$ the limits of current microwave technology. Extremely compact THz data to-point signal directionality, and 
therefore covertness, similar to that of a laser.

Our project objective was to explore THz technologies for wider bandwidth communications and sensing for DOD systems. More specifically the objective was to develop solid state THz sources for operation in the range between 0.3 to 10 ( 1 millimeter to 30 micrometer) mainly for aerospace communications and atmospheric sensing; and potentially, short-range terrestrial and airborne communications and near-object analysis. The focus was on all solid state high efficiency, high power, directional sources or emitters.

The exploration of the $\mathrm{THz}$ region relies heavily on the availability of coherent sources of $\mathrm{THz}$ radiation. However, there is a gap in commercial solid state sources. Conventional electronic sources are limited by resistances, capacitances, and transit times, resulting in significant attenuation of high frequency power. As one approaches $\mathrm{THz}$ frequencies, the output power of optical sources are limited by slow radiative processes. This project investigated recent advances in a type of sweep oscillator based on photonic mixing that has the greatest tuning range of any known coherent source in the $\mathrm{THz}$ region, and has the additional benefits of being all-solid-state and operating without any cryogens, high voltages, or large magnetic fields. 


\section{Publications}

\subsection{Manuscripts submitted, but not published}

a. "Bright THz Luminescence by Down-Conversion of Amplified-SpontaneousEmission”, E.R. Brown, J. E. Bjarnason, T.L.J. Chan, and A.W.M. Lee

\subsection{Papers published in peer-reviewed journals}

a. "Ultrafast photoresponse at $1.55 \mu \mathrm{m}$ in InGaAs with embedded semimetallic ErAs nanoparticlesm” D. C. Driscoll, M. P. Hanson, A. C. Gossard, and E. R. Brown, Appl. Phys. Lett. 86, 51908 (31 Jan 2005)

b. "Room Temperature, THz Photomixing Sweep-Oscillator and its Application to Spectroscopic Transmission through Organic Materials”, E. R. Brown, J. Bjarnason, T.L.J. Chan*, M.A. Celis, D. C. Driscoll, M. Hanson, and A. C. Gossard, Rev. Scientific Inst., vol. 75, pp. 5333-5342 (2004).

c. "ErAs:GaAs photomixer with two-decade tunability and $12 \mu \mathrm{W}$ peak output power,” J.E.Bjarnason, T.L.J.Chan, A.W.M.Lee, E.R.Brown, D.C.Driscoll, M.Hanson, A.C.Gossard, and R.E.Muller, Appl. Phys. Lett. vol. 85, pp. 3983-3985 (2004).

d. "Attenuation Contrast between Biomolecular and Inorganic Materials at THz Frequencies”, T. L. J. Chan, J. E. Bjarnason, A. W. M. Lee, M. A. Celis, and E. R. Brown, Appl. Phys. Lett. 85, 2523 (2004)

e. "MM-Wave, THz, and Mid-Infrared Transmission through Common Clothing” J. E. Bjarnason, T. L. J. Chan, A. W. M. Lee, M. A. Celis, and E. R. Brown, Appl. Phys. Lett. 85 (4) 519 (2004)

f. “A System-level analysis of Schottky diodes for incoherent THz imaging arrays”, E.R. Brown, Article Solid-State Electronics, Volume 48, Issues 10-11, OctoberNovember 2004, Pages 2051-2053

g. "Optical attenuation signatures of Bacillus subtillis in the $\mathrm{THz}$ region”, E. R. Brown, J.E.Bjarnason, T.L.J. Chan, A.W.M. Lee, and M.A. Celis, Appl. Phys. Lett. 84 (18) 3438 (2004)

h. "Picosecond photocarrier-lifetime in ErAs:InGaAs at $1.55 \mu \mathrm{m}$," M. Sukhotin, E. R. Brown, D. Driscoll, M. Hanson, and A. C. Gossard, Appl. Phys. Lett., vol. 83, pp. 3921-3923 (2003).

i. “Terahertz Generation by Photomixing in Ultrafast Photoconductors,” E.R. Brown, Int. J. High Speed Circuits and Systems, Vol. 13, No. 2, pp. 497-546 (2003).

j. "Photomixing and photoconductor measurements on ErAs/InGaAs at $1.55 \mu \mathrm{m}$ " M. Sukhotin, E. R. Brown, A. C. Gossard, D. Driscoll, M. Hanson, P. Maker, and R. Muller, Appl. Phys. Lett., Vol. 82, pp. 3116-3118 (2003). 
k. “A new design for increased THz power from LTG GaAs photomixers,” D. J. Yeh and E. R. Brown, SPIE Proc. on Terahertz and Gigahertz Photonics and Electronics, Vol. 4111, p. 124-32 (2000).

l. "A photoconductive model for superior low-temperature-grown $\mathrm{GaAs} \mathrm{THz}$ photomixers,” E. R. Brown, Appl. Phys. Lett., vol.75 (6), p.769-71 (1999).

\subsection{Recent Book Chapters}

a. "Fundamentals of Terrestrial Millimeter-Wave and THz Remote Sensing,” E.R. Brown, in Terahertz Sensing Technology, Vol. 2: Emerging Scientific Applications and Novel Device Concepts, ed. by D.L. Woolard, W. R Loerop, and M.S. Shur (World Scientific, Singapore, 2004)

b. "Terahertz Generation by Photomixing in Ultrafast Photoconductors,” E.R. Brown, in Terahertz Sensing Technology, Vol. 1: Electronic Devices and Advanced Systems Technology, ed. by D.L. Woolard, W. R Loerop, and M.S. Shur (World Scientific, Singapore, 2003).

\subsection{Papers presented at meetings, but not published in conference proceedings}

a. "THz Spectroscopy and Detection of Bioparticles and Biomaterials”, E.R. Brown, T.L. Chan, J.E. Bjarnason, A.W.M. Lee, SPIE Optoelectronics, January 25, 2004

b. THz Photomixing Sweep-Oscillator and its Application through Biomaterials",

E.R. Brown, T.L. Chan, J.E. Bjarnason, A.W. M. Lee, SPIE, Optoelectronics, January 25, 2004

c. “THz and Ultrafast Electronics”, E. Brown, J. Jensen, L. Kish, ECOG Workshop, 2004

\section{Accomplishments}

\subsection{Background on Coherent Sources}

An all-solid-state continuous-wave sweep oscillator has been developed providing high-resolution, transmission measurements between $\sim 20 \mathrm{GHz}$ and 3 THz. It is based on difference-frequency generation between two cw frequencyoffset lasers driving an ultrafast photoconductive mixer (photomixer). The output 
power around $100 \mathrm{GHz}$ is approximately $10 \mu \mathrm{W}$, falling to about $1 \mu \mathrm{W}$ around $1 \mathrm{THz}$ and $0.1 \mu \mathrm{W}$ around $3 \mathrm{THz}$. The sweep oscillator is used with two types of detectors: a hot electron bolometer for high-sensitivity measurements of weak absorption features below $1 \mathrm{THz}$, and a room temperature Golay cell for course measurements anywhere between $20 \mathrm{GHz}$ and $3 \mathrm{THz}$. The sweep oscillator facilitates the rapid characterization of a broad variety of materials including inorganic solids, biological materials, liquids, and gases with far greater resolution ( $10 \mathrm{MHz})$, frequency accuracy $(\sim 0.1 \mathrm{GHz})$, and spectral density $(\sim 1$ $\mu \mathrm{W} / \mathrm{MHz}$ ) than competitive wideband instruments such as Fourier-transform or time-domain spectrometers. To demonstrate the versatility of the sweep oscillator, results are presented for two diverse cases of interest: (1) fast scan, broadband (> $1 \mathrm{THz}$ ) absorption profiles from biomaterials such as polysaccharides, and (2) slow scan, narrow-band ( 1 GHz) atmospheric lines from water vapor.

Fig. 1 summarizes the present status of tunable, coherent THz sources; the most powerful being fundamental oscillators such as free-electron lasers, and certain types of vacuum tubes (e.g., backward-wave oscillators or BWOs). Free-electron lasers easily provide enough tunability and output power ( $>1 \mathrm{~mW}$ average) for most laboratory sciences (e.g., molecular chemistry), but are very large and expensive. Some vacuum tube devices (e.g., backward wave oscillators) are far smaller and less expensive but are still generally not considered suitable for portable systems because of their need for high voltage, high magnetic fields, or both.

As in other spectral regions, the most desirable sources are usually those based on 
solid-state devices. On the long-wavelength end of the THz region up to about $300 \mathrm{GHz}$, fundamental-frequency oscillators have been developed including InP-Gunn and siliconIMPATT diodes. While these sources have demonstrated impressive output power $(\geq 1$ $\mathrm{mW}$ ) up to $\sim 300 \mathrm{GHz}$, none of them have demonstrated the tunability generally required to do broadband $\mathrm{THz}$ spectroscopy. In addition the output power above $300 \mathrm{GHz}$ drops very quickly as the frequency approaches the maximum oscillation frequency $\left(f_{\max }\right)$, which is generally around $1 \mathrm{THz}$ at best.

At the upper end of the $\mathrm{THz}$ region ( 3 to $10 \mathrm{THz}$ ) photonic techniques are quite promising, particularly quantum-cascade lasers (QCLs) based on intersubband transitions of electrons in multiple-quantum-well structures. ${ }^{1,2}$ QCLs have recently displayed continuous-operation (at cryogenic temperatures) to $\lambda>80 \mu \mathrm{m} .{ }^{3,4}$ As in mid-IR semiconductor-based diode lasers, the challenge is to realize room-temperature operation.

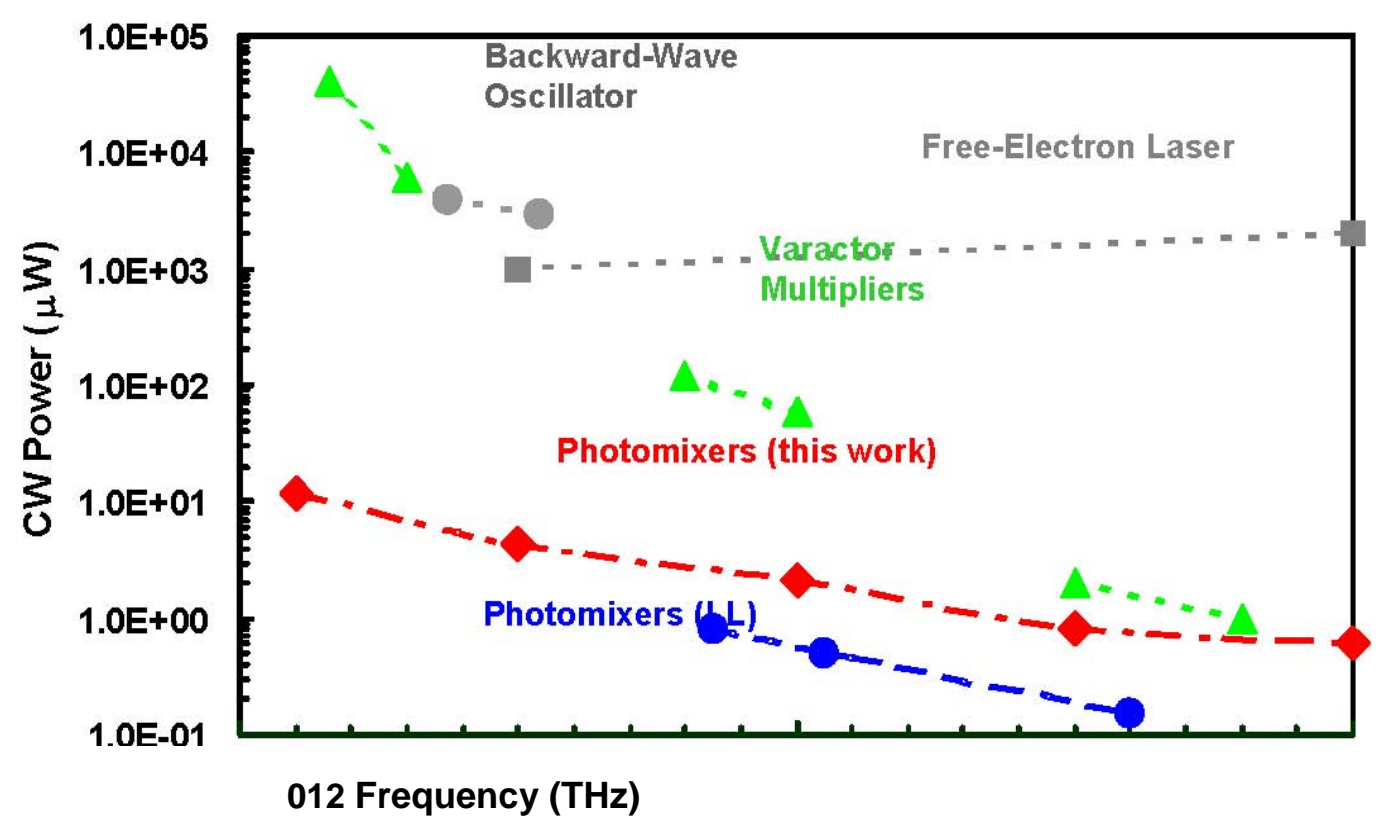

Fig. 1 Approximate average output power of various THz sources in use today. 
Such lasers are often limited in performance by nonradiative relaxation mechanisms when the photon energy becomes comparable to the energy quanta of lattice vibrations (i.e., phonons), as occurs at the meV levels of $\mathrm{THz}$ photons. Reducing the device temperature alleviates this problem, but only up to the point where defect or carriercarrier scattering (which are weakly dependent on temperature) takes over, and at the practical cost of cryogenic operation. Consequently, the gain medium must be embedded in a high-Q resonator, which then makes it difficult, if not impossible, to tune over a wide range.

To avoid the problems faced by fundamental sources, researchers have sought to produce tunable, coherent power in the THz region by harmonic multiplication of fundamental sources from the long-wavelength end, or by nonlinear-optical (e.g., threewave) mixing from the short-wave end. ${ }^{5}$ Of the two techniques, harmonic multipliers have been more successful and are now the most common solid-state coherent source up to frequencies around $1 \mathrm{THz}$. They provide the highest output power, although tunability is usually limited. ${ }^{6,7}$ The state-of-the-art in solid-state THz harmonic multipliers is shown in Fig. 1. The output power of multipliers continues to increase because of improvements in both the Schottky diodes and the drive devices and circuits. For example, MMIC solid-state power amplifiers now operate up to about $100 \mathrm{GHz}$ and can be configured to drive the first stage of varactor multiplier chains with power levels up to $100 \mathrm{~mW} .^{8}$ Three-wave mixing entails the injection of two frequency-offset laser beams in to a nonlinear-optical material such as LiNbO3. ${ }^{9}$ Because of the substantial $\chi^{(2)}$ susceptibility in the material, the two beams will produce a third beam at the difference frequency provided that all three have the same phase velocity. Unfortunately, three- 
wave mixing has never produced useful THz power levels in spite of the use of powerful drive lasers. The problems stem from the difficulty in achieving precise phase matching and in the fundamental limitation on the conversion efficiency imposed by the ManleyRowe relation, which states that at most one photon can be produced at $\left|\omega_{2}-\omega_{1}\right|$ for one photon each at $\omega_{1}$ and $\omega_{2}$. In other words, the best that three-wave mixing can ever do in terms of optical-to-electrical (O-E) conversion efficiency, e, is $\sim\left|\omega_{2}-\omega_{1}\right| / 2 \omega_{1}$, which is $\sim 0.2 \%$ in going from $1 \mu \mathrm{m}$ to $300 \mu \mathrm{m}$.

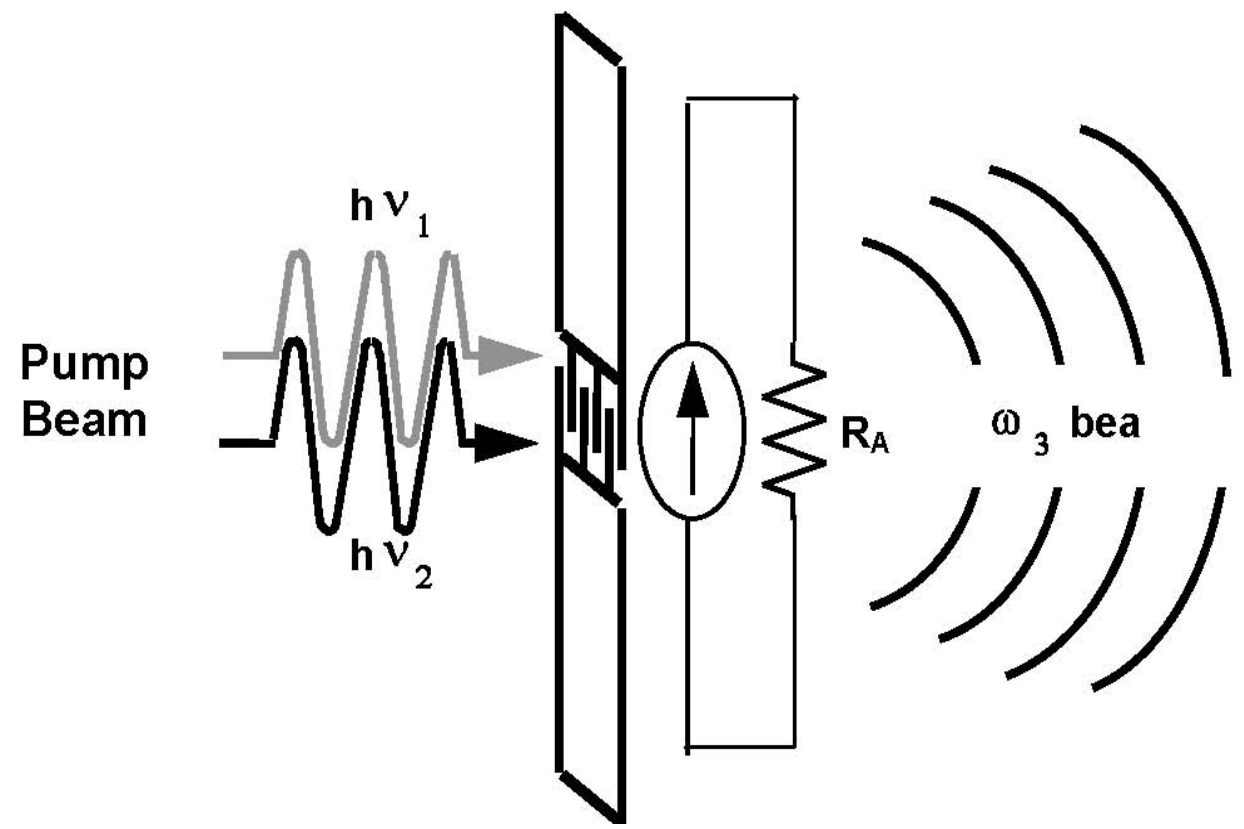

Fig. 2 Schematic diagram of interdigitated photomixer coupled to a strip line antenna, and simplified equivalent circuit in terms of a $\mathrm{THz}$ current source coupled to an antenna of radiation resistance $\mathrm{RA}$.

\subsection{Coherent THz Power by Photomixing}

A similar and alternative technique to three-wave mixing is photoconductive mixing, or photomixing for short. As shown in Fig. 2, photomixing entails the pumping of a high-speed photoconductive diode with two frequency-offset lasers. The best "ultrafast” photoconductive diodes have been made with low-temperature-grown GaAs 
or ErAs: GaAs, both of which can display very short electron-hole excess lifetimes ( $\sim 25 \mathrm{ps}$ ) and high electric breakdown field $\left(>5 \times 10^{5} \mathrm{~V} / \mathrm{cm}\right) .{ }^{10,11}$ Compared to older ultrafast photoconductive materials such as defective silicon, the LTG GaAs and ErAs:GaAs provide subpicosecond lifetime while maintaining useful levels of photocarrier mobility (> $100 \mathrm{~cm}^{2} / \mathrm{V}$-s for electrons and holes).

\section{Optical Drive}

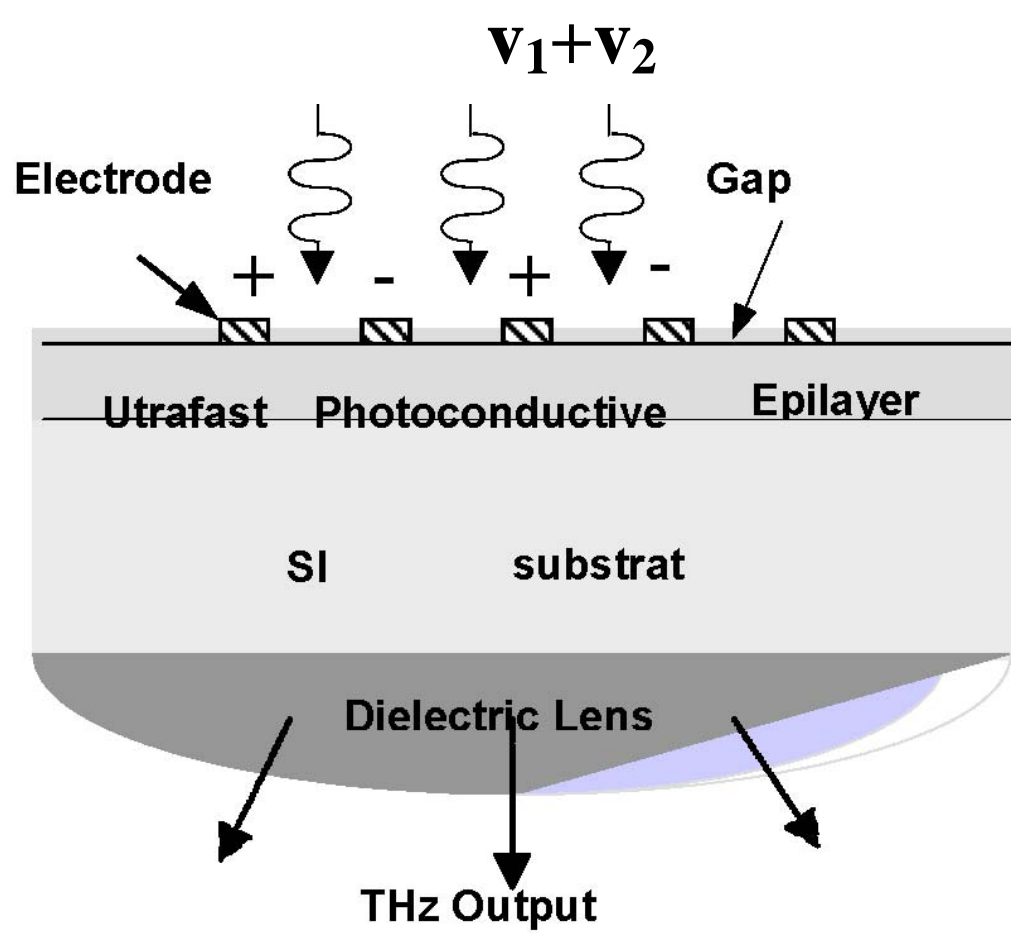

Fig. 3 Cross-sectional view of interdigitated-electrode photomixer illuminated from the air side by two frequency-offset laser beams.

Like other photoconductive devices containing homogeneous semiconductors, the photomixer must be biased with an internal electric field using electrodes such as those in the interdigitated structure shown in Fig. 3. Lasers are then used to illuminate the gap region between adjacent electrodes. If the laser photon energy is greater than the band gap of the photoconductive semiconductor, then most of the light will be absorbed via the 
internal photoelectric effect whereby one photon generates one electron-hole pair. If the absorption length is comparable to the gap width, then most of the electron-hole pairs will be generated in regions of high applied electric field near the electrodes. The resulting drift of these carriers to the attractive electrode will, according to the Shockley-Ramo theorem, induce current in the external circuit.

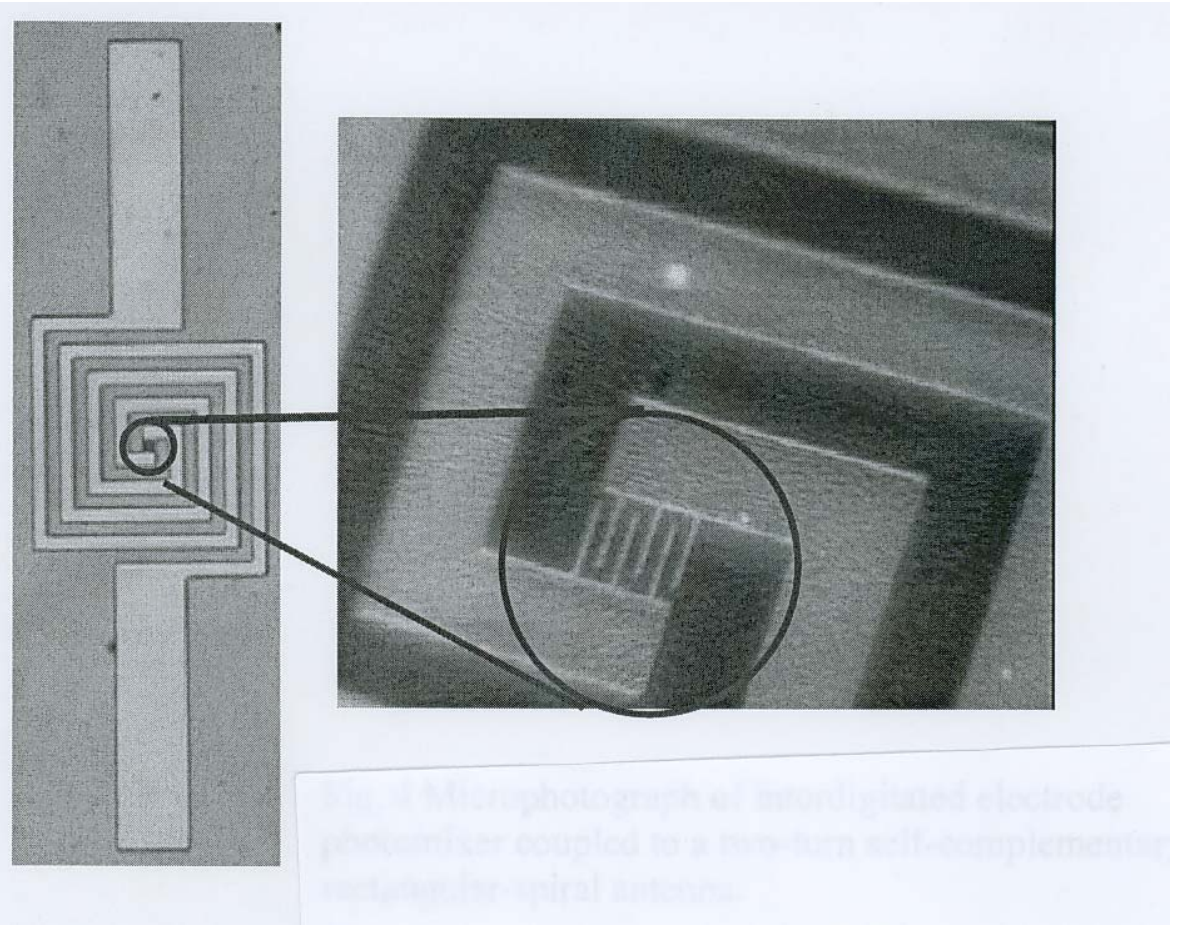

Fig.4 Microphotograph of interdigitated electrode photomixer coupled to a two-turn selfcomplementary rectangular-spiral antenna.

The key to THz generation by photomixing is the fact that the photoelectric effect is inherently quadratic with respect to the electric field and, therefore, mixes the optical fields via a product term. A fraction of the photocarrier concentration then oscillates at the difference frequency, which generates an ac current in the presence of the dc bias. The difference-frequency current generates $\mathrm{THz}$ power by connecting the diode to a suitable load, such as the planar self-complementary rectangular spiral shown in Fig. 4. 
A second key to $\mathrm{THz}$ photomixing is the availability of single-frequency widely tunable lasers made from either solid-state or semiconductor gain materials. During the past decade, solid-state lasers based on Ti:Al2O3 and erbium-doped fiber have been developed to operate at a single frequency over a very wide tuning bandwidth ( $10 \mathrm{~nm}$ or more) with high levels of cw output power (> $1 \mathrm{~W}$ ). Semiconductor diode lasers have proven more challenging but have been successfully developed with single-frequency operation using various optical techniques such as distributed Bragg reflectors and external gratings, and output power $>100 \mathrm{~mW}$.

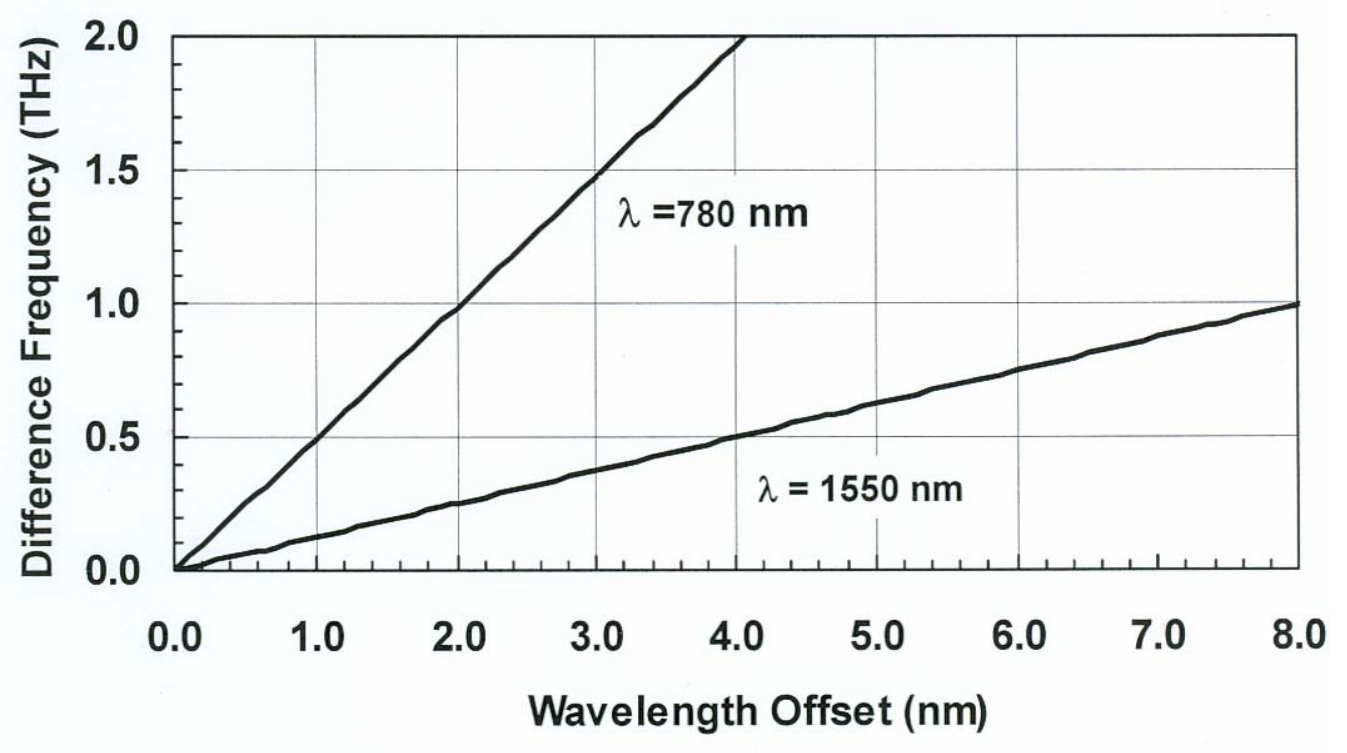

Fig. 5 Photomixing frequency curve for pump lasers centered at 780 and $1550 \mathrm{~nm}$. 


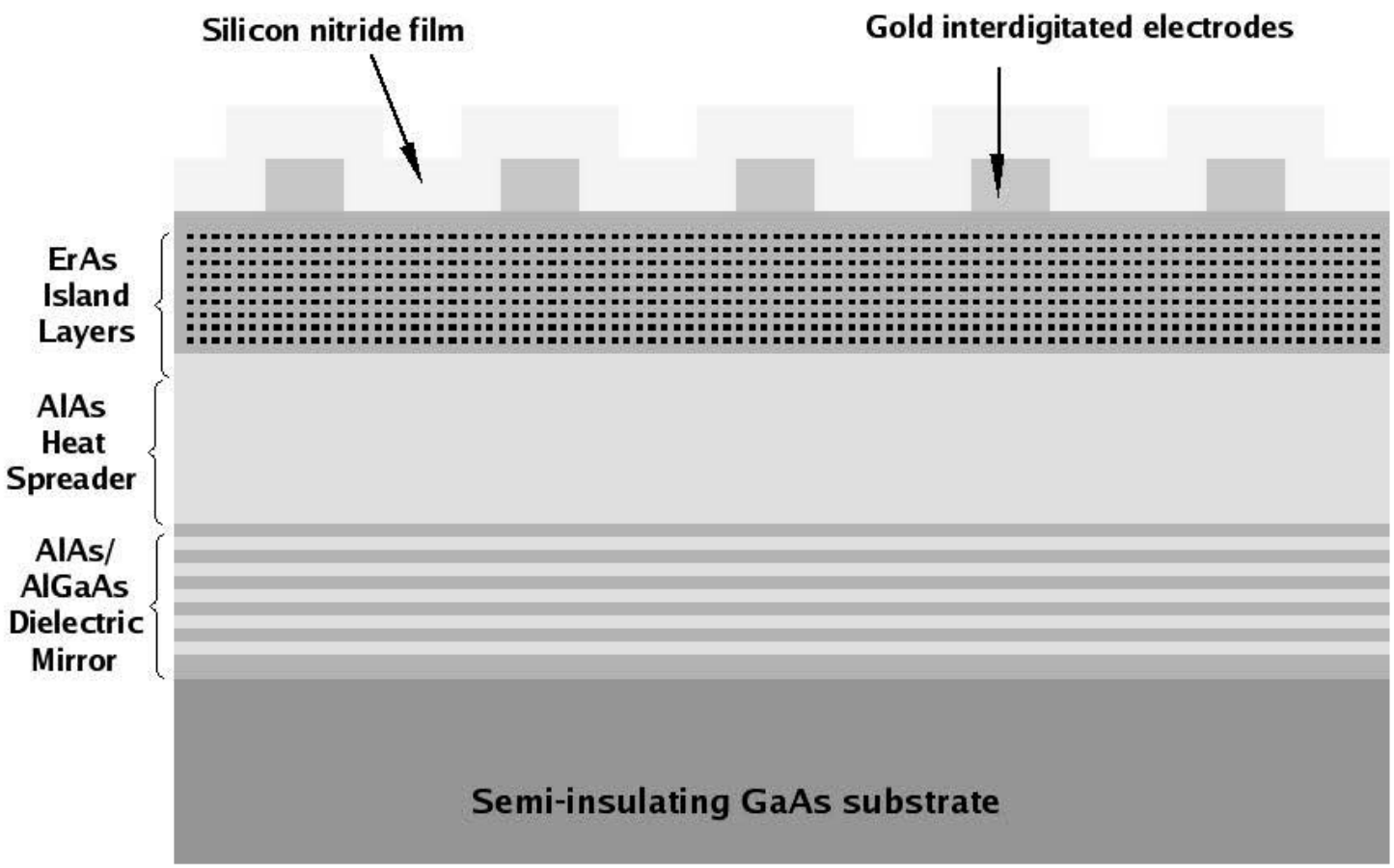

Fig. 6 Cross section of photomixer device

\subsubsection{Photomixing and photoconductor measurements on ErAs/GaAs at $780 \mu \mathrm{m}$}

We report the fabrication and demonstration of an ErAs:GaAs interdigitated photomixer as a tunable $\mathrm{THz}$ source ranging from $\sim 20 \mathrm{GHz}$ to $\sim 2 \mathrm{THz}$, with a record high $12 \mu \mathrm{W}$ maximum power typically around $\sim 90 \mathrm{GHz}$. Each photomixer is coupled to a composite dipole-spiral planar antenna that emits a Gaussian-like beam into free space. The beam switches from dipole to spiral antenna behavior as the frequency increases. A distributed Bragg reflector is embedded in the device beneath the photomixer to increase its external quantum efficiency. The photomixer has a 900-Å-thick silicon nitride coating, which serves as an antireflection and passivation layer, and also improves the 
reliability and heat tolerance of the device.

A photomixer device was grown and fabricated on a semi-insulating GaAs substrate. Its cross section is shown in Fig. 6. The first epitaxial layer is an AlAs/AlGaAs distributed Bragg reflector (DBR), which reflects $760 \mathrm{~nm}$ light but passes THz radiation. The DBR consists of 12 interlaced 12.65- $\AA$ AlAs and 13.57- $\AA$ AlGaAs layers. The second epilayer is an AlAs heat spreader ${ }^{13}$ and the third one is an ErAs:GaAs layer made by embedding layers of ErAs into the GaAs during growth. The ErAs forms nanoparticles, which act as fast nonradiative recombination centers without degrading the mobility too much.

Interdigitated fingers and composite spiral-dipole antennas were imprinted onto the GaAs surface by defining their pattern in polymethylmethacrylate with electron beam lithography, followed by metalizing with 200 and 2000- $\AA$-thick Ti and Au films respectively. The 0.2 - $\mu \mathrm{m}$-wide interdigitated electrodes and spiral-dipole arms resulted from removal of the unwanted metallization by chemo-mechanical liftoff. Then, a 900- $\AA$-thick film of silicon nitride was deposited on the top surface to prevent the peeling of the interdigitated fingers during operation of the device. ${ }^{14}$ The nitride film thickness was also chosen to act as a quarter-wave antireflection coating for the incident laser power. In order to supply bias to the device, via holes were etched into the nitride at each end of the dipole, which was then bonded with gold wires. After extended use of the resulting photomixers, it has become clear that the nitride layer is additionally protecting and passivating the photomixer surface, particularly against environmental hazards such as dust particles.

Fig. 7 shows the block diagram of the THz setup in which the photomixer is 
biased by a DC power supply and driven by two frequency-offset master oscillator power amplifier (MOPA) lasers, one of which is tunable within a fraction of a GHz. Both lasers operate around $775 \mathrm{~nm}$ at room temperature. Their output beams are co-aligned and divided with a beam splitter so that one part is coupled through an optical fiber into an optical spectrum analyzer (OSA) and the other is focused on the photomixer after passing through an isolator

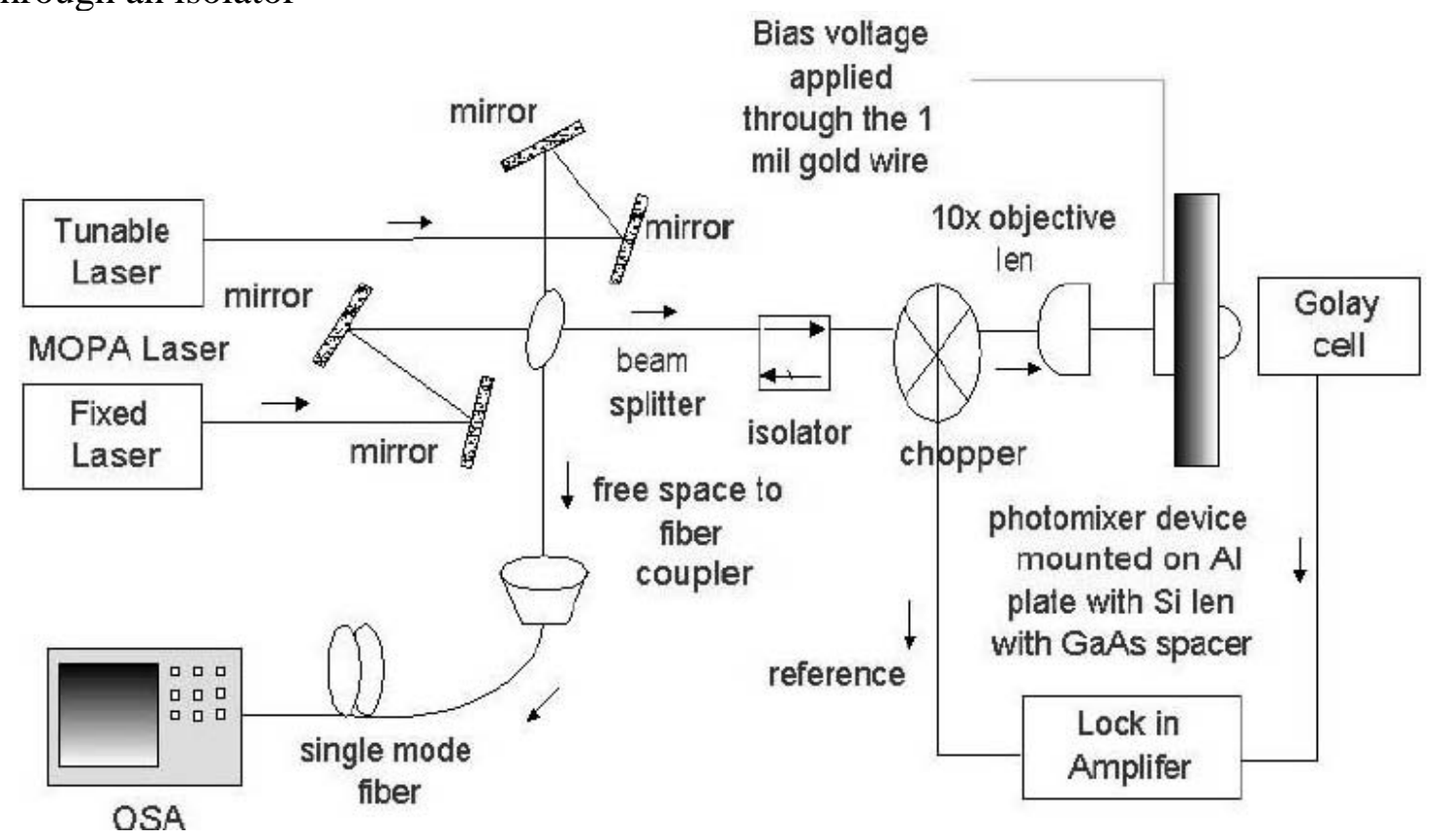

Fig. 7 Experimental setup used to characterize ErAs:GaAs photomixers.

to eliminate back-reflection into the lasers. The OSA allowed us to conveniently monitor the wavelength of both lasers without disturbing the photomixing experiment. The photomixing frequency, given theoretically by the frequency difference of the two lasers, is therefore known at any time. The laser power incident on the photomixer is measured by placing an optical power meter in front of the isolator.

The ac current generated by the photomixing drives a composite dipole-spiral 
antenna, which is coupled to free space via a hyperhemispherical Si lens. The output power of the photomixer is sensed with either a 6-mm-aperture Golay cell or a hot electron bolometer (HEB) depending on the purpose of the experiment. The HEB outperforms the Golay cell in terms of sensitivity and speed but the Golay cell's maneuverability was preferred when maximizing output power. One or two $0.25-\mathrm{mm}-$ thick sheets of black polyethylene were placed in front of the Golay cell to block ambient background radiation and thermal radiation from the photomixer caused by pump-laser heating. The Golay cell was calibrated at $104 \mathrm{GHz}$ with the heavily attenuated radiation from a Gunn diode. ${ }^{15}$ The unattenuated Gunn diode power was calibrated separately using a waveguide power meter. To improve sensitivity and reduce the effects of drift, the lasers were chopped at 25 or $50 \%$ duty cycle and the amplified output of the Golay cell was synchronously detected using a digital lock-in amplifier. 


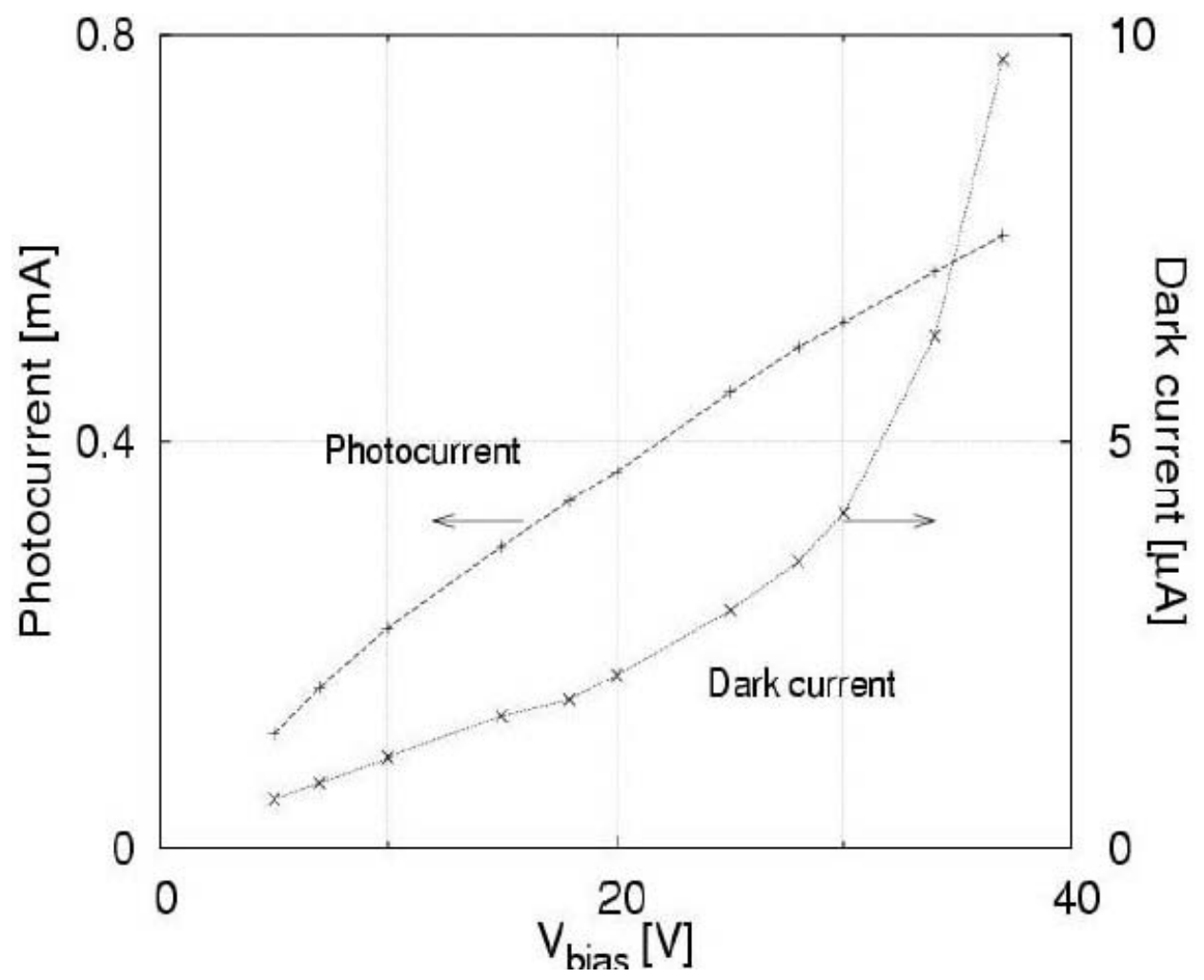

Fig.8 Current vs. Voltage in ErAs:GaAs photomixer. The dark current was measured without optical pumping (dark) and the photocurrent with a $30 \mathrm{~mW}$ optical power at $\Gamma$ (photocurrent).

The simplest means of qualifying the photomixer is by current-voltage (I-V)

measurements leading to the plots in Figs. 8 and 9. As seen from the dark current curve in Fig. 8, the zero- bias dark resistance of this device is $\mathrm{R} \approx 9 \mathrm{M} \Omega$. At around $30 \mathrm{~V}$ bias the dark current increases rapidly and soft breakdown occurs. Fig. 9 shows the I-V curve with the device illuminated by laser power levels of $20 \mathrm{~mW}$ and $40 \mathrm{~mW}$. On these curves, a cusp was observed around $30 \mathrm{~V}$ bias. This is the first observation of such a feature in a photomixer and is still being investigated. 


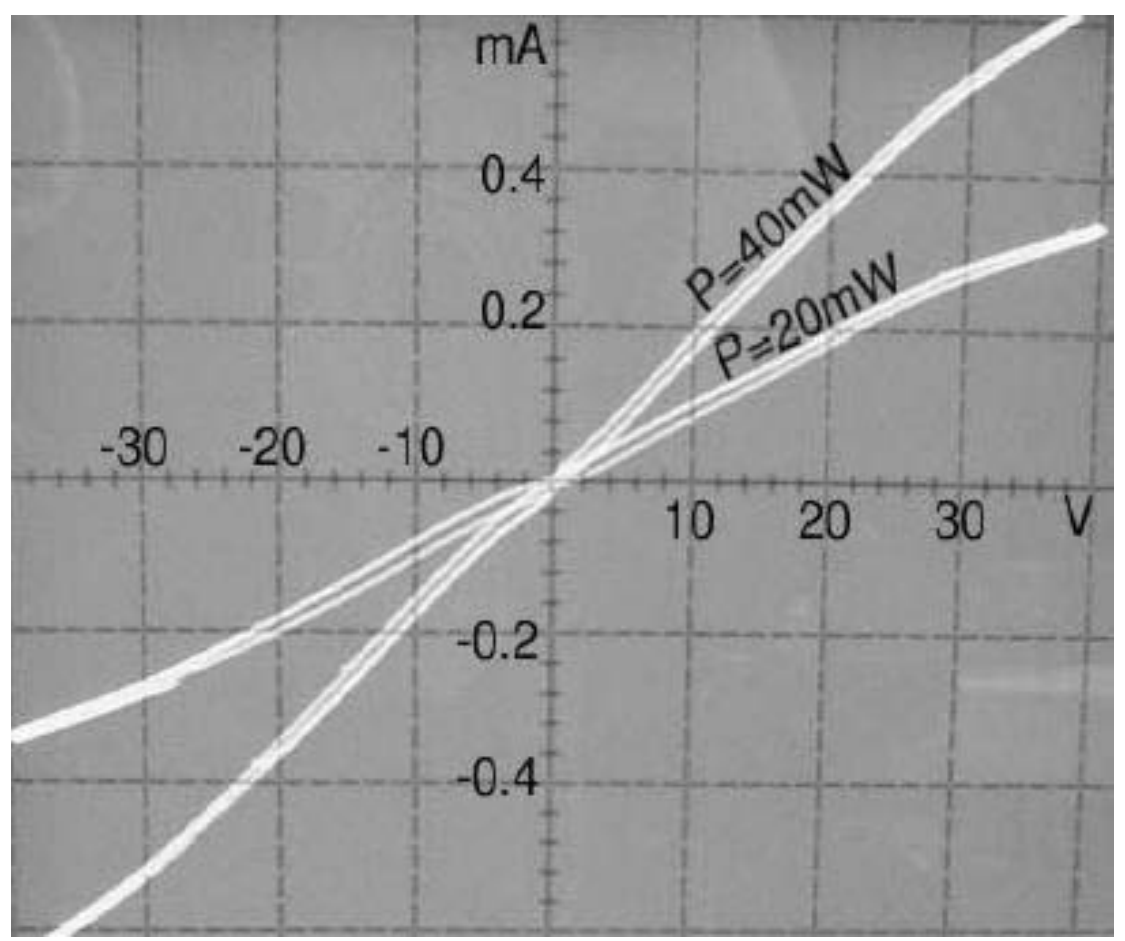

Fig. 9 Current vs. Voltage extracted from a curve tracer connected to ErAs:GaAs photomixer illuminated with 20 and $40 \mathrm{~mW}$ laser.

Fig. 10 shows the output power measured around $90 \mathrm{GHz}$ as a function of pump power over the range up to $60 \mathrm{~mW}$ per laser. The chopper duty cycle was reduced to $25 \%$ for this experiment to avoid damage by overheating. The maximum THz power measured from our photomixer before burning it out was $12 \mu \mathrm{W}$ at $88 \mathrm{GHz}$ with $60 \mathrm{~mW}$ optical power, $30 \mathrm{~V}$ bias, and $1.9 \mathrm{~mA}$ of average photocurrent. The frequency at which the maximum occurs is thought to be that which gets closest to a conjugate match between the photomixer and antenna impedance. To the authors' knowledge, $12 \mu \mathrm{W}$ is the highest output power reported from a $\mathrm{THz}$ photomixer to date at frequencies around $100 \mathrm{GHz}$ or above. 


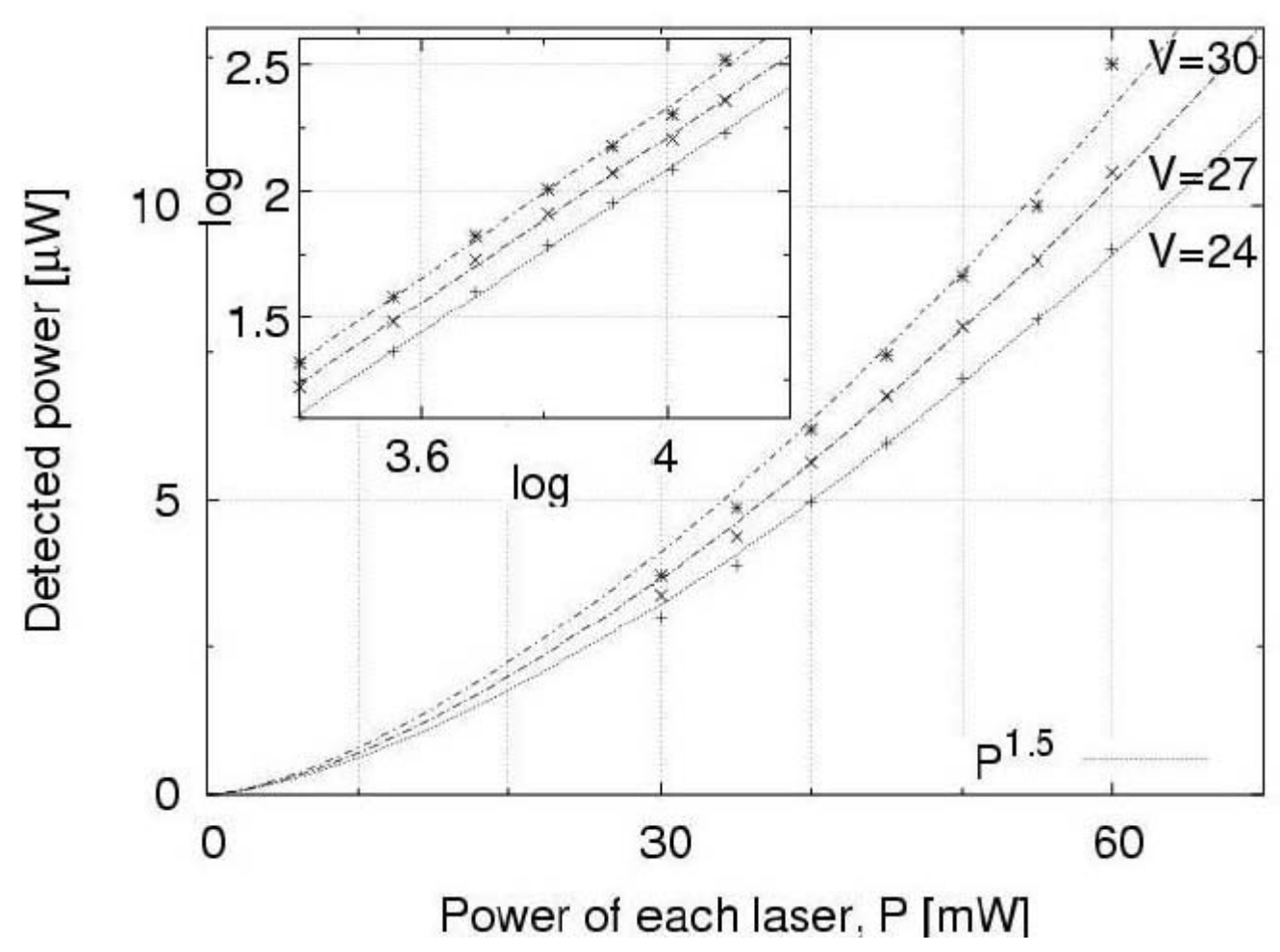

Fig.10 Output power of photomixer vs. Laser pump power under constant bias conditions ( $\mathrm{V}=24,27$ and 30) and offset frequency $\Delta \mathrm{f}=88$.

To explain the performance curves in Fig. 6, photomixing theory predicts the amplitude of the time varying part of the photocurrent to be

$$
i=\frac{2 \eta e}{h v}\left(P_{o p t, 1} P_{o x, 2}\right)^{v^{2}}{\frac{1}{1+(\Delta \omega \tau)^{2}}}^{1 / 2}
$$

where $P_{1}$ and $P_{2}$ are the input powers of each laser, $\Delta \omega$ is the frequency offset of the lasers, $h v$ is the energy quantum of each laser, $\eta$ is the external quantum efficiency and $\tau$ is the recombination lifetime. Assuming that the radiated power rises quadratically with current, the average output $\mathrm{THz}$ power is given by $\mathrm{P}_{\text {out }}=\mathrm{i}^{2} \mathrm{R}_{\mathrm{L}} / 2$ where $\mathrm{RL}$ is the radiation resistance of the antenna. Hence, the output power should be proportional to the power product of the lasers, $\mathrm{P}_{\text {out }} \propto$ 
$\mathrm{P}_{\text {opt }, 1} \cdot \mathrm{P}_{\mathrm{opt}, 2}$ or $\mathrm{P}_{\text {out }} \propto\left(\mathrm{P}_{\mathrm{opt}}\right)^{2}$ if the both lasers have the same power. The experimental results in Fig. 6 show that the output power is increasing slower than theory predicts. This can be attributed to reduction in the photocurrent cause by reduction of the average carrier velocity at the high junction temperatures attained in this experiment ( $>200^{\circ} \mathrm{C}$ ). Fig. 11 shows the typical output power spectrum of this photomixer as a function of difference frequency up to $2 \mathrm{THz}$. The spectrum was acquired by pumping the photomixer with 60 mW total optical power. The output power was measured with a 2 Golay cell having a polyethylene window and, therefore, lowers standing-wave ratio and better infrared background noise rejection than the diamond-window device. The Golay cell's 6-mm aperture limits the lowest detectable frequency to 20 $\mathrm{GHz}$, but alternative means of detecting the power are likely to show output power at even lower frequencies. At $1 \mathrm{THz}$ the output power of the present device is down approximately $10 \mathrm{~dB}$ below the $100-\mathrm{GHz}$ value. If the lowfrequency power is assumed to be $10 \mu \mathrm{W}$ and the responsivity of the Golay cell is assumed to be flat with frequency, then the THz output power exceeds $1 \mu \mathrm{W}$. This makes our present results around $1 \mathrm{THz}$ comparable to the best photomixer power levels reported to date; e.g., $3.0 \mu \mathrm{W}$ at $0.8 \mathrm{THz}, 2.0 \mu \mathrm{W}$ at $1.2 \mathrm{THz}$, and $0.8 \mu \mathrm{W}$ at $1.6 \mathrm{THz}$ from three separate resonant (twin-slot) antenna photomixers. $^{17}$ The output power approaching $2 \mathrm{THz}$ in Fig. 11 becomes comparable to the total infrared power, which the Golay cell detects from the heating of the device by the chopped lasers.

To better characterize the THz output beam, transmission measurements were 
made with a polarizer consisting of a grid of 0.10-mm-dia. parallel brass wires with 0.15 mm spacing, oriented either vertically or horizontally with respect to the axis of the dipole arms. The graph indicates that the $\mathrm{THz}$ radiation from the composite dipole-spiral antenna is polarized along the dipole axis in the low frequency $(<200 \mathrm{GHz})$ end, but circularly polarized at higher frequencies (> $200 \mathrm{GHz}$ ). This shift from linear to circular polarization is attributed to the dipole-spiral duality of the antenna. For a dipole of length $\mathrm{L}$, the largest peak in output power generally occurs at the first full-wave resonance. On a dielectric half-space, this occurs at a frequency f given by $L \approx \lambda_{\text {eff }}=\mathrm{c} /(\mathrm{f}$ $n_{\text {eff }}$ ) where $c$ is the speed of light in vacuum and $n_{\text {eff }}=\left[\left(1+\varepsilon_{\mathrm{GaAs}}\right) / 2\right]^{1 / 2} \approx 3.7$. For the present photomixer, the end-to-end length of the dipole arms is $540 \mu \mathrm{m}$. Using this length in the full-wave resonance expression, we find $\mathrm{f}=150 \mathrm{GHz}$. Much beyond this frequency, the dipole radiation should weaken and the spiral antenna begins to dominate the radiation resistance, consistent with the experiments. A room-temperature $\mathrm{THz}$ source with the power, tunability, and coherence of the photomixer reported in this paper has significant practical and scientific potential in applications such as THz spectroscopy, bioparticle detection, and imaging, to name a few. For example, a recent experiment using the photomixer to measure transmission spectra through different types of common fabrics indicates that imaging through most clothing is possible up to at least $500 \mathrm{GHz}$. 


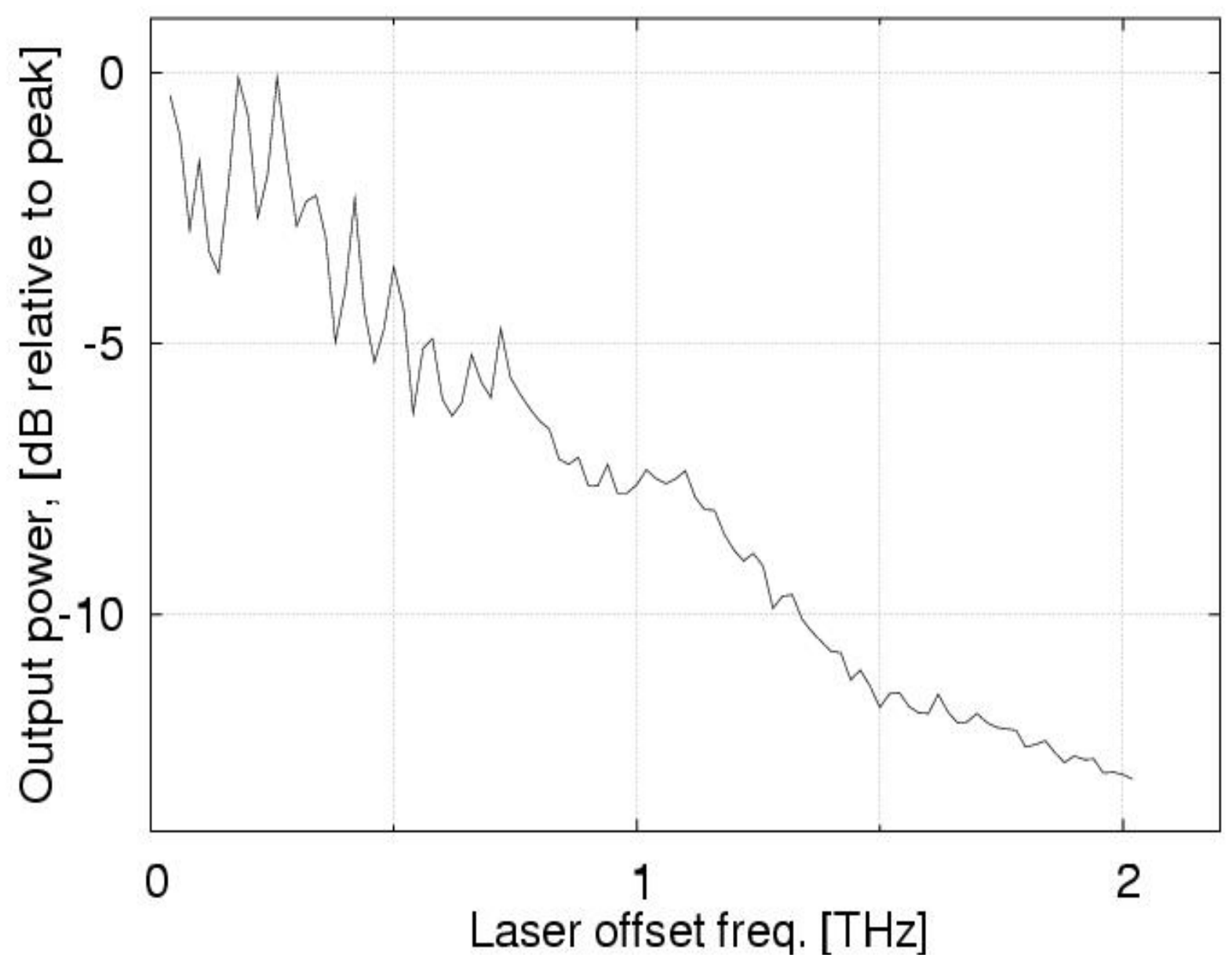

Fig. 11 Output power from a photomixer driven dipole-spiral antenna detected with a Golay cell vs. frequency offset of incident lasers.

\subsubsection{Photomixing and photoconductor measurements on ErAs/InGaAs at $1.55 \mu \mathrm{m}$}

We report here the first measurements of microwave and mm-wave photomixing in ErAs:In0.53Ga0.47As-on-InP at 1.55- $\mu \mathrm{m}$. In this, material 30 thin layers of ErAs nanoparticles are embedded in the InGaAs matrix $40 \mathrm{~nm}$ apart to form superlattice structure ${ }^{18}$ as shown in Fig. 12. 


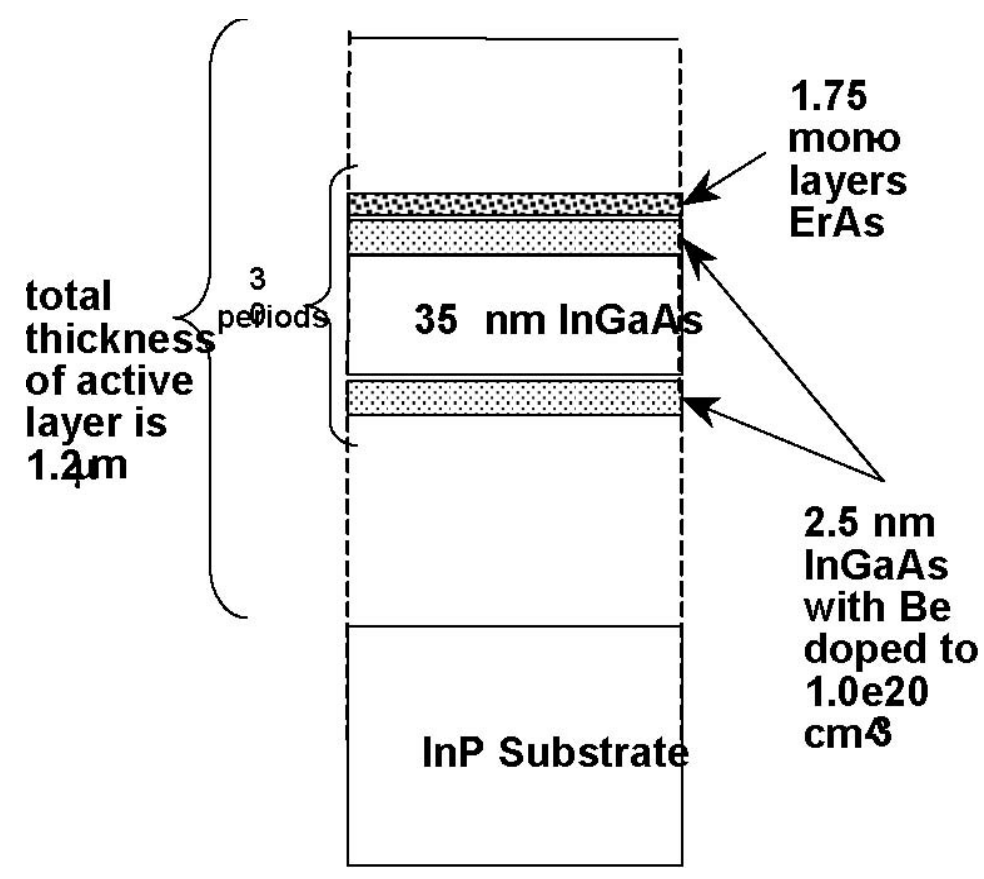

Fig.12 Material Structure: ErAs:GaAs

Interdigitated electrodes are deposited on the top surface to form the active area of the photomixer, which is coupled to a dipole antenna to radiate out the difference-frequency power into free space ${ }^{19,20}$, Electrical qualification of the photomixers was carried out by measurements of the I-V characteristics, resistivity, and breakdown field. The I-V curve shown in Fig. 13 is a good indicator of the (dark) resistance of the device, which was previously too low to make useful photomixers. Through advances at UCSB involving ErAs incorporation and Be compensation, the resistivity has been increased to useful levels. From the quasi-static capacitance of our interdigitated electrodes, $\mathrm{C} \sim \mathrm{fF}$, and the measured zero-bias resistance of our I-V curve, we find $\mathrm{RC}=5.4$ fs. From electrostatics, we can set this equal to the dielectric relaxation time $\tau$ through $\mathrm{CR}=\varepsilon / \sigma=\varepsilon$. $\rho$, leading to

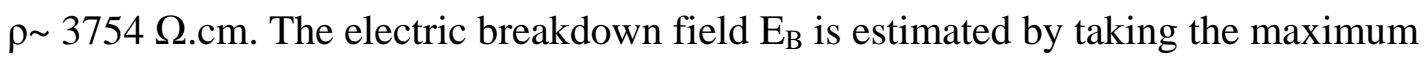
voltage bias the photomixer and dividing by separation between interdigitated fingers $(1 \mu \mathrm{m})$. 


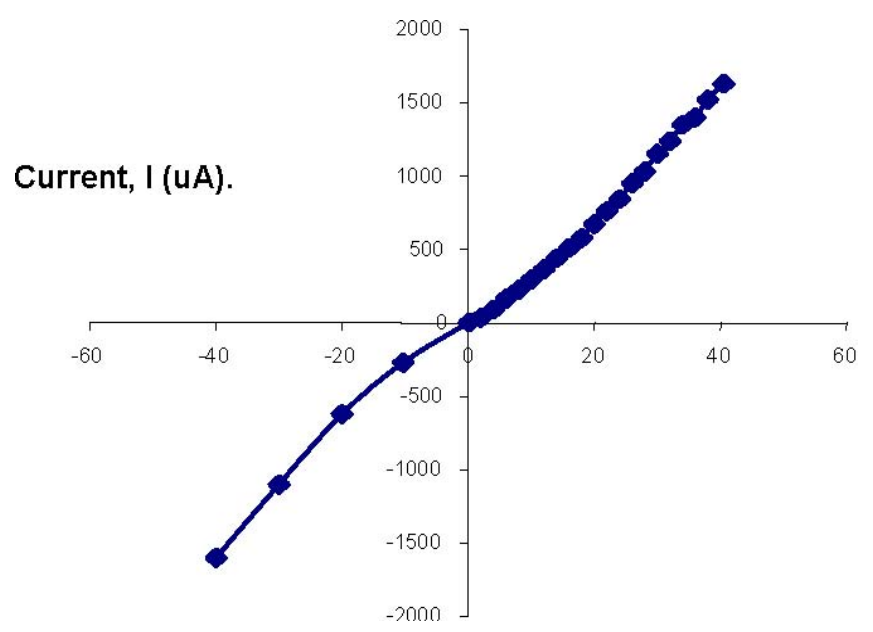

Biasing Voltage (Volts).

Fig.13 Plot of Current vs. Voltage in ErAs/InGaAs Photoconductor

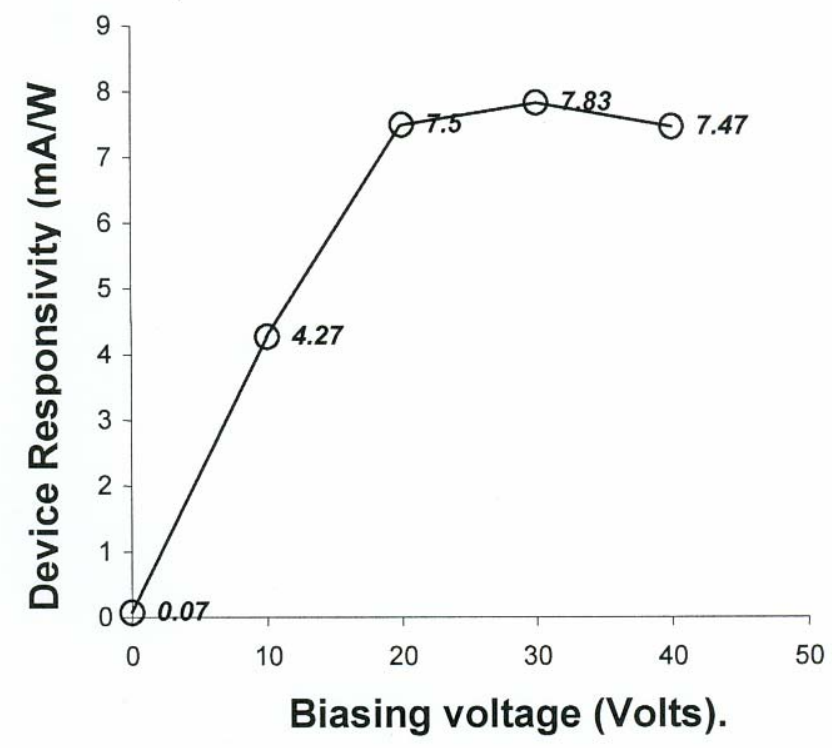

Fig. 14 Responsivity as a Function of Vbias

This results in $\mathrm{E}_{\mathrm{B}} \sim 4 * 10^{5} \mathrm{~V} / \mathrm{cm}$. Note that the choice of maximum bias is somewhat arbitrary since the breakdown in these devices is "soft", similar to the impact-ionization breakdown of low-temperature-grown GaAs. Optical qualification of the photomixers was made through measurements of the DC responsivity and optical-to-RF conversion. Results for the $1.55-\mu \mathrm{m}$ dc responsivity are shown in Figure 14 . At 30 volts bias, the 
responsivity saturated at a maximum around $7.8 \mathrm{~mA} / \mathrm{W}$. Photomixing experiments were performed using two frequency-offset external cavity diode lasers (ECDLs) at $1.55 \mu \mathrm{m}$. The two ECDL beams were mixed in fiber via a 3-db fiber coupler and amplified by an EDFA. A fiber-coupled lens was used to focus the beam from fiber through free space and on to the device. Up to $26 \mathrm{GHz}$, difference frequency power was measured by directly connecting the device terminals to a RF spectrum analyzer via a coaxial cable. For frequencies greater than $100 \mathrm{GHz}$, a Golay cell was used to detect the difference frequency power coupled off-chip by a silicon lens through free space.

Results of this measurement are shown in Figure 15.

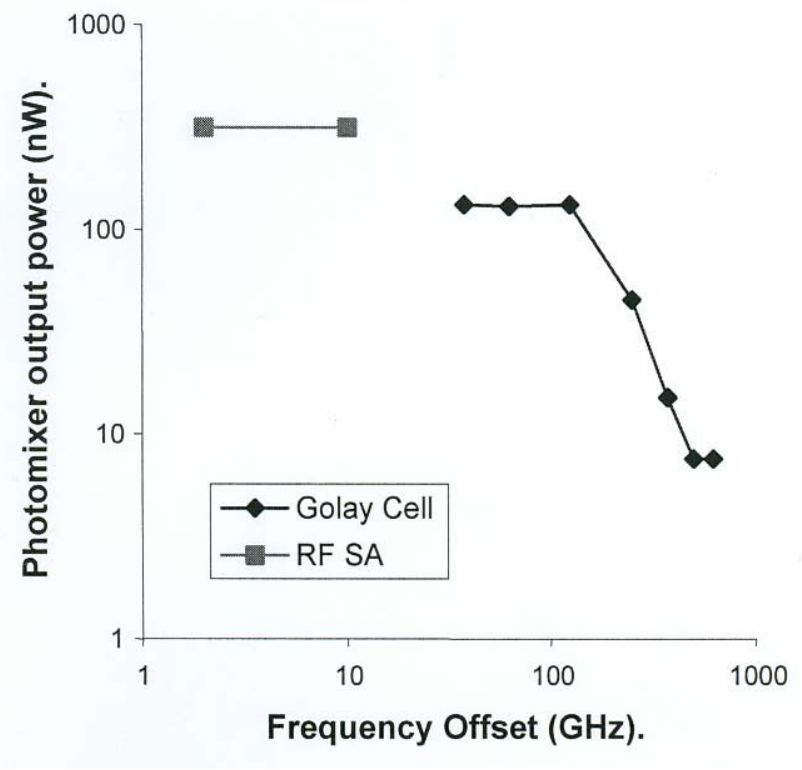

Fig.15 Power vs. Offset Frequency

The output power of $0.13 \mu \mathrm{W}$, is practically flat up to about $200 \mathrm{GHz}$ and then drops rapidly. Based on these measurements, the photocarrier lifetime of the material is $\approx 1.0$ ps. By reducing the separation between the ErAs nanoparticle layers in future 
photomixer layers, we expect to reduce this lifetime below 1 ps so that $\mathrm{THz}$ photomixers can be fabricated.

\subsection{Design of the THz Sweep Oscillator}

The broad tunability of the photomixer lends itself naturally to the role of an electronically controlled sweep oscillator - a building block for many RF instruments and systems, such as scalar and vector network analyzers. A sweep oscillator is also very useful for wideband spectroscopy, and photomixers have already been utilized for gas molecular spectroscopy in two different modes: (1) frequency-locked, limited scan mode

using a high-sensitivity (liquid-helium cooled) detector, ${ }^{22}$ and (2) unlocked, coherent mode where a second photomixer is used as a homodyne receiver by driving it with the same two frequency-offset lasers as the transmit photomixer. ${ }^{23}$ The present sweeposcillator design is different than the previous two in that it can cover two full decades of tunability all under electronic (laptop computer) control. And it does so with semiconductor-based pump lasers and a simple room- temperature direct detector (Golay cell).

\subsection{Photomixing Diode and Planar Antenna}

The photomixer used for the present sweep oscillator consists of six 0.2-micronwide interdigitated electrodes separated by five 1.6-micron gaps and occupying a total area of $9.2 \times 9.2$ microns. These dimensions are a result of several engineering trade-offs discussed at length elsewhere. If the gaps are made much smaller to achieve high photoconductive gain, the capacitance gets large and limits the power and O-E efficiency 
at sub- $\mathrm{THz}$ frequencies through $\mathrm{RC}$ rolloff, where $\mathrm{R}$ is antenna radiation resistance. If the gaps are made much larger to reduce the capacitance, the inverse transit time and associated photoconductive gain g decay, limiting the output power and O-E efficiency at moderate drive power levels. If the area is made much larger to allow for more optical pump power, the capacitance increases and causes RC roll off well below $1 \mathrm{THz}$. If the area is made much smaller, burnout invariably occurs because of the high junction temperature that results from the combination of optical drive and electrical bias.

As shown in Fig. 1, the output power of photomixers coupled to planar antennas has previously been limited to a few $\mu \mathrm{W}$ below $1 \mathrm{THz}$, and progressively lowers values at frequencies above $1 \mathrm{THz}$. So in the present sweep oscillator design, three improvements were added to the basic photomixer design: (1) a resonant-optical-cavity (ROC), (2) an AlAs heat spreader, and (3) a silicon-nitride protective cap layer. ${ }^{24}$ The details of these design improvements are discussed elsewhere.

Besides its planar geometry and low specific capacitance, another benefit of the interdigitated-electrode photomixer is that it creates a balanced current feed for simple planar antennas such as dipoles and slots. No balun or similar circuit is required. Hence the photomixer can be fabricated in the driving gap of the antenna or coupled to the gap through a short transformer, which helps to keep the transmission-line lengths short and the THz resistive losses acceptably low. These facts have been utilized to develop a variety of planar-antenna photomixers based on logarithmic-spiral, twin-dipole, and twinslot antennas.

In the present sweep-oscillator, the photodiode is coupled to the driving point of a two-turn rectangular spiral antenna. As true for all self-complementary planar 
antennas, the rectangular spiral displays special electromagnetic characteristics, namely a driving-point impedance that is almost purely real and independent of frequency over a wide operational bandwidth (determined by the inner turn and outer turn radii). For the two-turn spiral shown in Fig. 4, this bandwidth can be one decade or more. The value of the driving-point resistance is given by $\mathrm{RA}_{\mathrm{A}}=60 \pi /\left(\varepsilon_{\mathrm{eff}}\right)^{1 / 2}$ where $\varepsilon_{\mathrm{eff}}$ is the effective dielectric constant. For a planar antenna on a dielectric half-space, $\varepsilon_{\text {eff }}$ can be approximated by $\left(1+\varepsilon_{\mathrm{r}}\right) / 2$. For GaAs, $\varepsilon_{\mathrm{r}} \approx 13.0$ so that $\mathrm{R}_{\mathrm{A}}=72 \Omega$. Further details of the rectangular spiral antenna performance will be presented elsewhere.

\subsection{Optical coupling to free space and to the sample}

Independent of the antenna type, a significant problem with any antenna-coupled device at $\mathrm{THz}$ frequencies is coupling out the radiation to free space. Given the dielectric constant of GaAs and the other semiconductors used to make photomixers and most other $\mathrm{THz}$ devices, the majority of radiation from the antenna tends to propagate into the substrate and get trapped there by total internal reflection. A clever way to prevent total internal reflection is through implementation of a back-side hemispheric lens made out of a high- $\varepsilon$ material, as shown schematically in Fig. 3. ${ }^{30}$ A good choice for the lens material is a low-cost, high-resistivity semiconductor, such as float-zone-grown silicon. If the photomixer antenna is located at the center of curvature of the lens and the lens is thick enough so that the spherical surface is in the far-field of the antenna, then all the radiation from the antenna reaches the spherical surface at normal incidence and passes in to free space with a reflection coefficient of $\left(n_{L}-1\right)^{2} /\left(n_{L}+1\right)^{2}$. This is a great improvement over the slab substrate, but yields a highly diverging beam from planar antennas having large 
beam solid angle. Thus, a common practice is to locate the antenna behind the center of curvature at a point where the radiation will be refracted in the forward direction. If the hemispherical surface and setback are fabricated in the same dielectric material, the resulting optic element is called a hyperhemispherical lens.
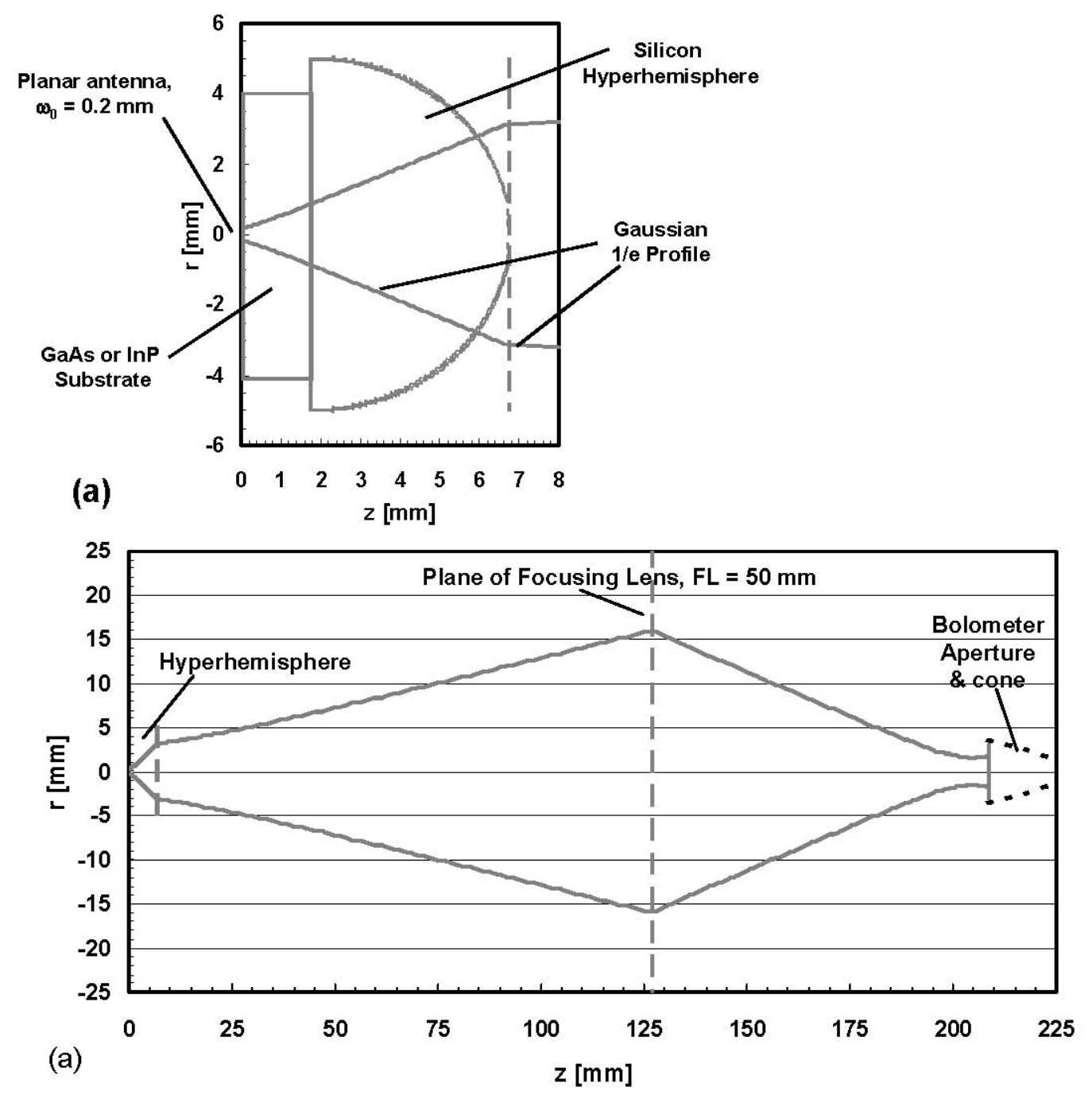

Fig. 16(a) Cross sectional view of photomixer chip coupled to silicon hyperhemisphere. 16(b) Gaussian-beam propagation diagram showing the 1/e intensity locus and the longitudinal position of all the optical elements between the photomixer and the bolometer power meter.

If $n_{\mathrm{L}}$ is perfectly matched to $\mathrm{n}_{\mathrm{S}}$ and the antenna is located behind the center of 
curvature by a distance $r / n$, then the lens focusing is aplanatic. This is a property of geometric optics which states that all rays from a given point in the object plane (in this case, the planar antenna) are refracted to a quasi-parallel bundle after the lens. Clearly, most planar antennas cannot be accurately analyzed by geometric optics. A treatment of the focusing problem by modal (Gaussian-beam) analysis, as displayed in Fig. 16(a), indicates that the optimum setback is just short of $\mathrm{r} / \mathrm{n}$. Once the radiation is coupled to free space, there are two options to couple the radiation to the sample: (1) Gaussian-beam matching, and (2) light-pipe coupling. In the Gaussian-beam approach of Fig. 16(b), the diverging beam from the photomixer is focused down to a beam waist by a plastic lens located at some distance from the hyperhemisphere. An important design issue is where the waist occurs and how big the minimum spot size will be. As shown by the two curved loci drawn through the $r=\omega$ points, the beam propagates through the GaAs substrate, into the Si hyperhemisphere, and is then transformed into free space using an ABCD matrix appropriate to a spherical-dielectric interface. ${ }^{32}$ To avoid significant totalinternal reflection, the hyperhemisphere can provide only a slight transformation of the beam, which remains diverging after passage through the Si-air interface. The free-space Gaussian beam then propagates to the (plastic) plano-convex lens which transforms the diverging Gaussian beam to a converging beam through the application of the thin-lens ABCD matrix. ${ }^{19}$ The waist shown in Fig. 16(b) has a minimum spot size $\omega_{0} \approx 1.5 \mathrm{~mm}$, consistent with the given frequency (300 GHz), the planar- antenna spot size $(\sim 0.2 \mathrm{~mm})$, the radius and set-back of the Si hyperhemisphere (5.0 and $1.76 \mathrm{~cm}$, respectively), and the focal length and diameter of the plastic lens (2.0 and 2.0 inch, respectively). The other important distances and dimensions are listed in Table 1. 
Table 1. Distance and dimensions in Gaussian matching

\begin{tabular}{|l|l|}
\hline Parameter & Value \\
\hline Free space wavelength [mm] & 1 \\
\hline Spot radius at spiral ant [mm] & 0.2 \\
\hline Index of hyperhemisphere & 3.435 \\
\hline Radius of hyperhemisphere [mm] & 5 \\
\hline Set-back of hyperhemisphere [mm] & 1.76 \\
\hline Focal length of lens [m] & 50 \\
\hline Hyper-to-lens separation & 120 \\
\hline Index outside hyperhemishpere & 1 \\
\hline Lens radius [mm] & 25.4 \\
\hline Lens to bolometer [mm] & 82 \\
\hline Bolometer aperture radius [mm] & 3 \\
\hline
\end{tabular}

The alternative technique for coupling the $\mathrm{THz}$ radiation to the sample holder is the lightpipe method shown in the block-diagram of Fig. 17. A 10-cm length of 0.5-inch innerdiameter brass tube is abutted to the hyperhemisphere at one end and to the sample holder at the other. An identical section of brass tube couples the output port of the sample holder to the one of the receivers. This technique takes advantage of the high reflectivity of polished brass in the THz region - a fact well known since early research in the 1960s. Experience indicates that the overall power coupling efficiency is comparable to the Gaussian set-up. The advantage of light-pipe coupling is spatial incoherence, better known as "beam scrambling." This is advantageous when measuring samples having high refractive index or in the form of etalons. Such samples tend to produce internal multiple-pass interference (i.e., standing waves), which complicate the interpretation of absorption features. With a scrambled beam, the broad spectrum of spatial frequencies 
making up the incident beam each resonate at different frequencies owing to different angles of propagation and, therefore, different path lengths through the sample.

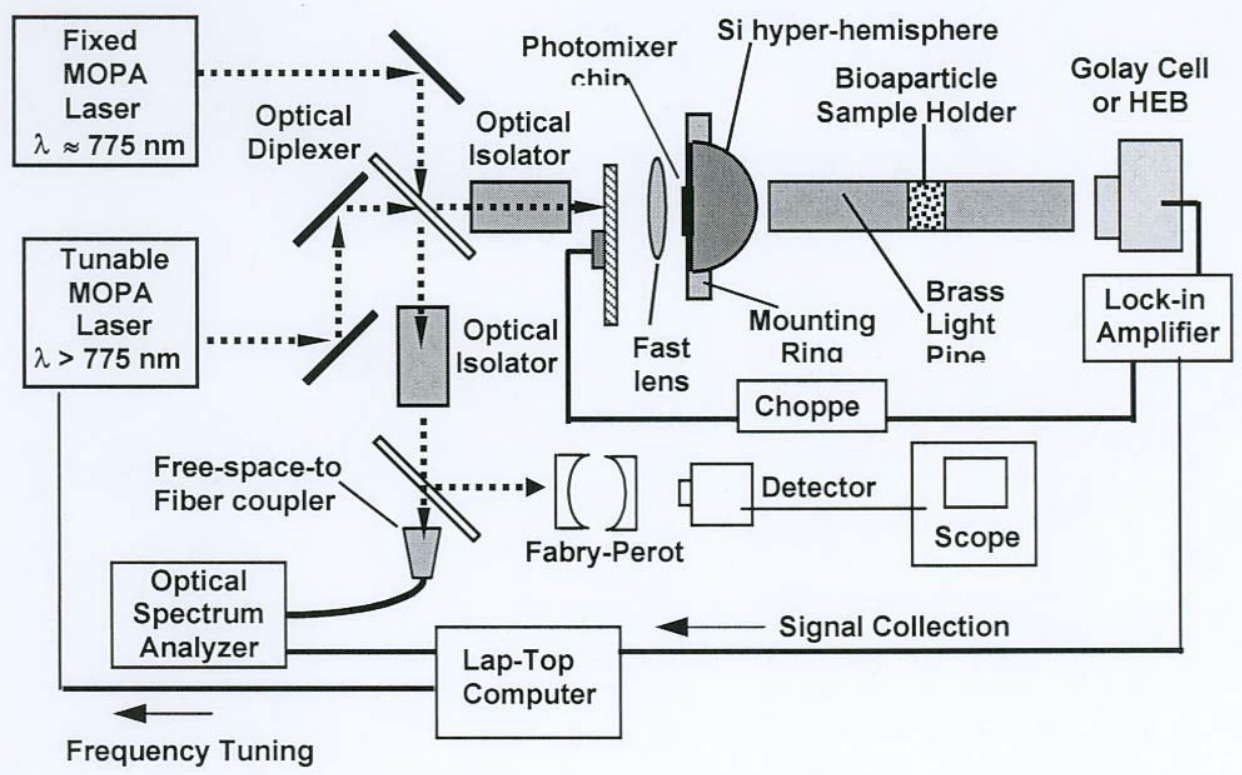

Fig. 17 Block diagram of the key components of the photomixing spectrometer.

\subsection{Receivers: Golay Cell and hot-electron Bolometer}

Perhaps the best choice for detecting the ultra wideband output from the sweep oscillator are receivers based on thermal detectors - devices that absorb the free-space radiation in a material or medium that is thermally isolated from the environment and transforms the temperature change into a significant change of some other physical parameter, be it optical or electrical. The dominant source of noise in such detectors is often thermal (i.e., Johnson-Nyquist) fluctuations, so reducing the device temperature liquid-helium (4.2 K) levels or less almost always increases the signal-to-noise ratio dramatically and reduces the noise-equivalent power (NEP) commensurately. The penalty for this improvement is, of course, cryogenic operation.

Both a room temperature and cryogenic detector have been developed for the 
sweep oscillator, the room-temperature device designed for broadband, low dynamic range scans and the cryogenic designed for narrow-band, high dynamic range scans. The room-temperature detector is the Golay cell - a venerable device that combines absorption of radiation by a thin metallic film and transfer of the heat into a gas. The expansion of the gas moves a membrane on which is mounted an optical grating or similar component. The motion of the grating diffracts light between a source and a photodetector, causing a large change in output voltage of the detector. In the authors' experience, the Golay cell is acceptably uniform in spectral response, having about 3-dB variations in optical response down to a long-wavelength cutoff determined by the diameter of the entrance aperture. The present Golay cell has a $6 \mathrm{~mm}$ aperture, yielding a long-wavelength cutoff (TE11 mode of circular waveguide) of $34 \mathrm{GHz}$. For frequencies around $100 \mathrm{GHz}$ and higher, the specific NEP is $\sim 2 \times 10^{-10} \mathrm{~W}-\mathrm{Hz}{ }^{-1 / 2}$. For the typical postdetection bandwidth of $20 \mathrm{~Hz}$, the minimum detectable power is thus $\sim 1 \mathrm{nW}$.

The maximum input power to the Golay cell is determined by saturation of the transduction mechanism. For the present Golay cell this power is roughly $\mathrm{P}_{\max } \approx 10 \mu \mathrm{W}$. The dynamic range is then given by $\mathrm{DR}=\mathrm{P}_{\max } /\left[\operatorname{NEP}\left(\tau_{\text {int }}\right)^{-1 / 2}\right]$ where $\tau_{\text {int }}$ is the postdetection integration time defined by the lock-in amplifier (Fig. 7). Hence, for the typical $\tau_{\text {int }}$ of $0.3 \mathrm{~s}$, the dynamic range is found to be $\mathrm{DR} \approx 3 \times 10^{4}$.

Our cryogenic thermal detector is an InSb hot electron bolometer (HEB) - an old mm-wave and THz detector with one key advantage over the Golay cell, which is speed. Like all bolometers, the HEB operates by having the absorbed power change the temperature of a "thermistor": a device that turns the absorption of $\mathrm{THz}$ power into a commensurate change of resistance. In the HEB, the "thermistor" is 
the electron gas of the InSb semiconductor which, because of its low concentration and mass, has a relatively short thermal time constant of $\sim 1 \mu$ s. As shown in Fig. 15, the HEB consists of a rod of highly pure and compensated InSb mounted across a circular-waveguide integrating cavity. The diameter of the oxygen-free copper waveguide is 0.090 inch corresponding to a cutoff frequency of the fundamental TE11 mode of $78 \mathrm{GHz}$. The HEB is mounted at the throat of a brass conical feed horn having a flare angle of $7.5^{\circ}$ and a length of 0.75 inch. At frequencies for which the feed horn is unimodal, the antenna pattern of the feed horn is asymmetric in the $\mathrm{E}$ and $\mathrm{H}$ planes but has small enough sidelobes to be useful. As the frequency approaches $425 \mathrm{GHz}$, the feed horn becomes highly overmoded but the antenna pattern remains because detector couples to most of the modes, particularly the TE ones.

The InSb HEB has long been one of the more popular detector types because of its low noise, insensitivity to infrared, and high-speed response compared to other bolometer types. Both the radiation absorption and the bolometric conversion are carried out by the free electron gas, which becomes strongly decoupled from the lattice at low temperatures. Unfortunately, its absorption coefficient drops rapidly with frequency above about $300 \mathrm{GHz}$ because in this range $\omega \tau_{\mathrm{S}}>1$ where $\tau_{\mathrm{S}}$ is the scattering time. 


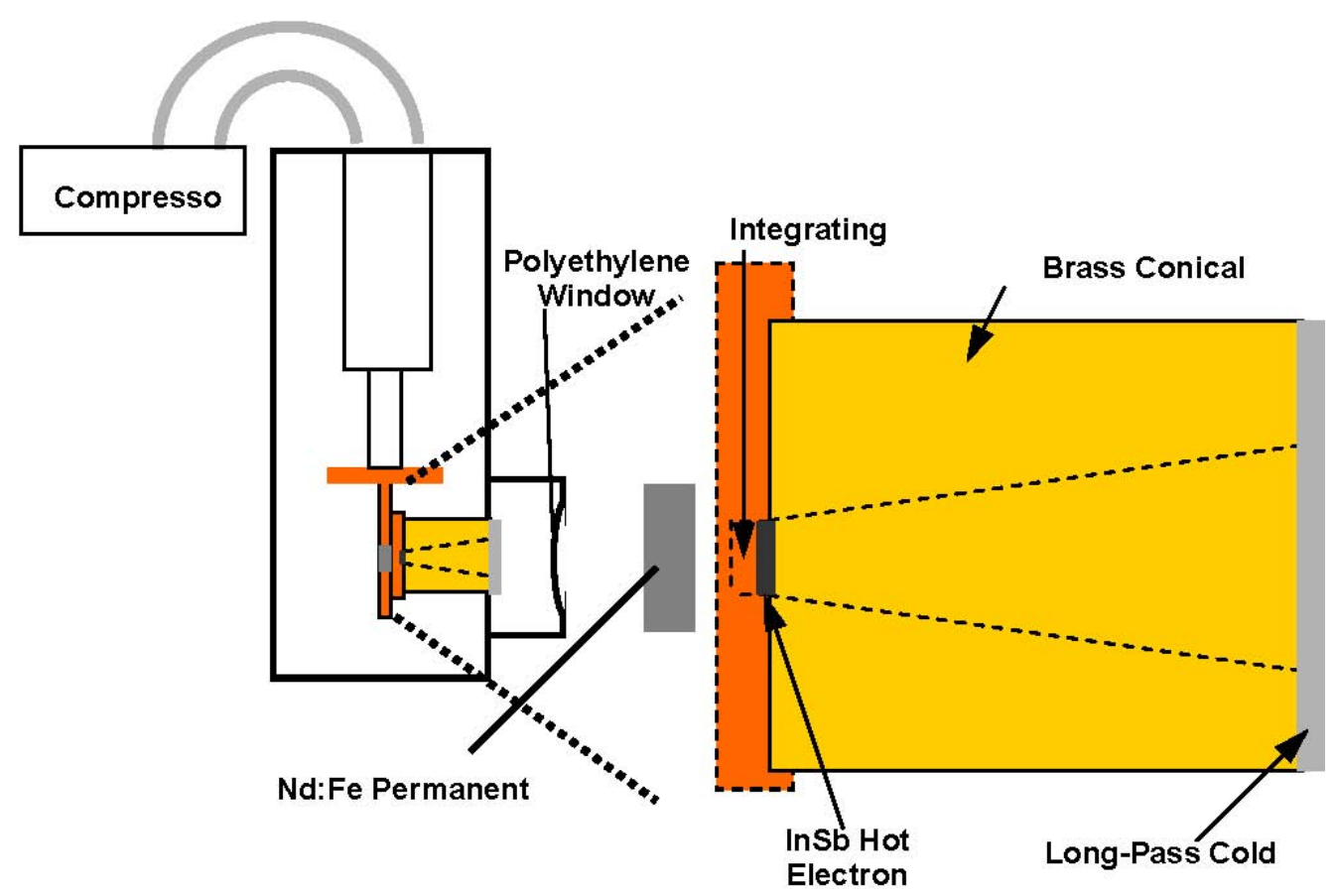

Fig. 18 Cryogenic detector apparatus used in consisting of an magnetically-enhanced InSb hot-electron bolometer cooled by a helium closed-cycle refrigerator and coupled to free space with a brass conical feedhorn.

An effective way to restore the strong absorption in this range is to apply a quantizing magnetic field. In our HEB receiver it was most convenient to use permanent magnets (Nd: Fe) having a remnant induction of about $10.5 \mathrm{KG}$. The magnets were placed in a round hole fabricated in the mounting block just behind the integrating cavity. We estimate that the magnetic induction that exists about $2 \mathrm{~mm}$ away at the InSb element is approximately 3 KG. From the known effective mass of InSb $\left(0.014 \mathrm{~m}_{0}\right)$ and the wellknown expression $\omega_{C}=\mathrm{eB} / \mathrm{m}^{*}$, we find a cyclotron resonance relation $\omega_{\mathrm{C}} \approx 200$ $\mathrm{GHz} / \mathrm{KG}$, so that at $\mathrm{B}=3 \mathrm{KG} \omega_{\mathrm{C}} \approx 600 \mathrm{GHz}$.

We have taken advantage of the HEB speed to make a low-temperature detector, high-sensitivity receiver without cryogens. The HEB is mounted in a (0.1 W dissipation) 
closed-cycle two-stage He ${ }^{4}$ Gifford-McMahon (GM) refrigerator as shown in Fig. 18.

Given the low HEB bias power and radiative loading, the cold-finger temperature readily drops to about $3.5 \mathrm{~K}$ and maintains this temperature for an indefinite time. The key drawback of the GM operation is the $\sim 0.1 \mathrm{~K}$ fluctuations that occur with each compressor cycle (0.7 s). Historically, this has precluded the application of bolometers because even 0.1-K fluctuations will usually cause deleterious variations in the responsivity. In our design, we chop the $\mathrm{THz}$ radiation as high as practical, $1.5 \mathrm{KHz}$, well above the spectrum of temperature fluctuations caused by the refrigerator. The HEB output is detected with a band-limited amplifier having a passband between 1.0 and $10 \mathrm{KHz}$ (Fig. 19). The amplifier is based on a super-low-noise operational amplifier (Analog Devices 797) having noise spectral densities of $\mathrm{v}_{\mathrm{n}} \approx 1.1 \mathrm{nV} / \mathrm{Hz}^{1 / 2}$, and $\mathrm{i}_{\mathrm{n}}=1 \mathrm{pA} / \mathrm{Hz}^{1 / 2}$. The differential resistance of the HEB at the operating point is $\approx 0.7 \mathrm{k} \Omega$, close to the "noise match" resistance $=\mathrm{v}_{\mathrm{n}} / \mathrm{i}_{\mathrm{n}}$ of the amplifier. 


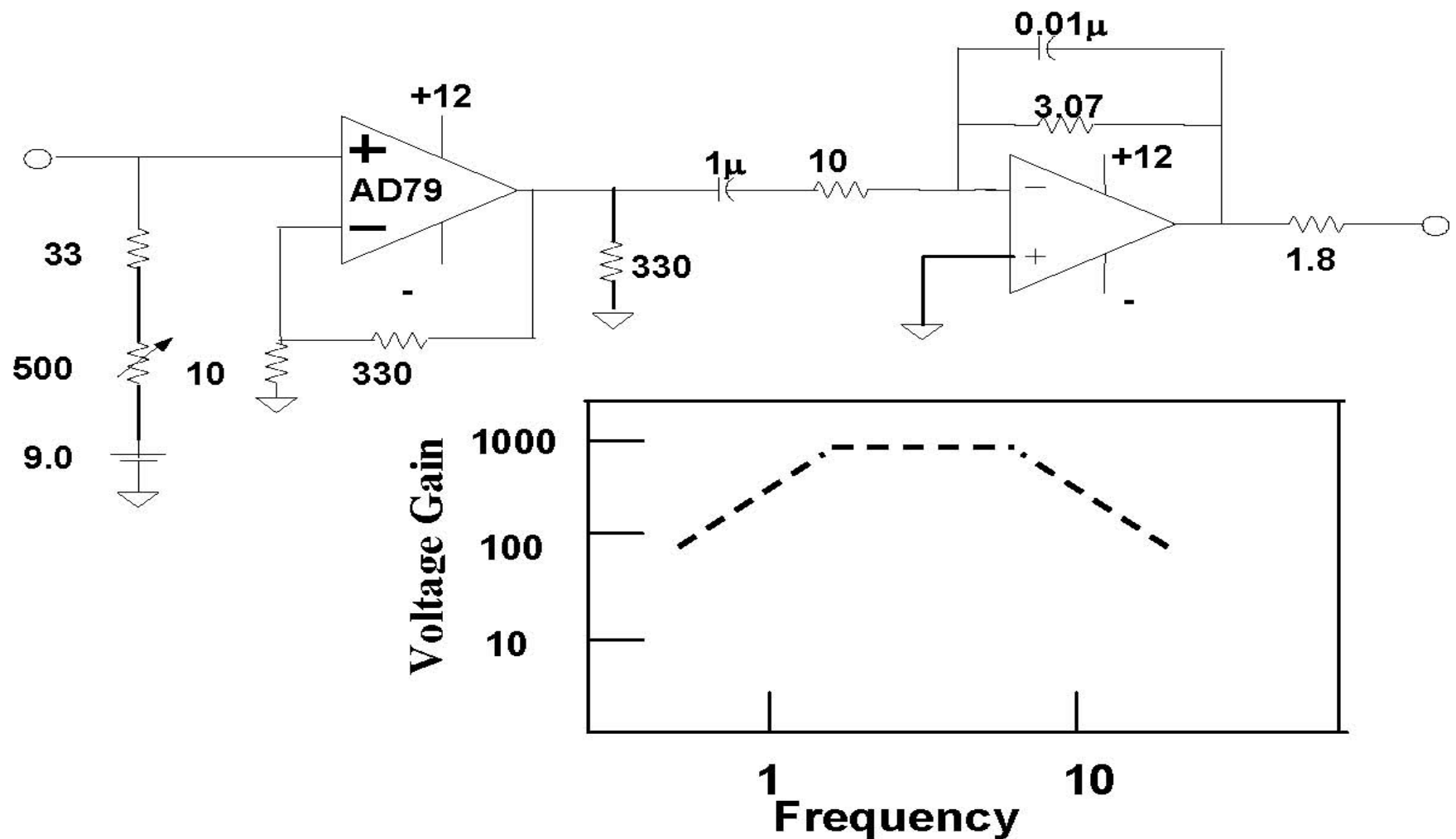

Fig. 19 Schematic diagram of low-noise amplifier used with hot-electron bolometer that provides an input noise voltage of $\sim 1 \mathrm{nV} / \mathrm{Hz}^{1 / 2}$.

Calibration of the HEB receiver was carried out without magnetic field using a Gunn oscillator at $104 \mathrm{GHz}$. ${ }^{34}$ The Gunn power was coupled to the InSb HEB through a standard gain pyramidal feedhorn and plastic coupling lenses. After correcting for the lens losses, the measured external responsivity was approximately $2100 \mathrm{~V} / \mathrm{W}$ - a value 3 times lower then the theory but not bad considering the possible beam mismatch losses, refrigerator window loss, etc. With this responsivity, the receiver $\mathrm{NEP}$ is $\approx 1 \times 10^{-12} \mathrm{~W} / \mathrm{Hz}^{1 / 2}$ - approximately two orders of magnitude more sensitive than the Golay cell.

The maximum input power to the HEB is determined by overheating of the electron gas to the point where the resistance-changes begin to disappear. For the present HEB this power is given by $\mathrm{P}_{\max } \approx 1 \mu \mathrm{W}$. The dynamic range is then given by $\mathrm{DR}=$ 
$\mathrm{P}_{\max } /\left[\mathrm{NEP}\left(\tau_{\mathrm{int}}\right)^{-1 / 2}\right]$ where $\tau_{\text {int }}$ is the post-detection integration time defined by the lock-in amplifier (Fig. 1). Hence, for the typical $\tau_{\text {int }}$ of $0.3 \mathrm{~s}$, the dynamic range is found to be $\mathrm{DR} \approx 5.5 \times 10^{5}$, a factor of 20 higher than the Golay cell in spite of the HEB's lower power-handling capability.

\subsection{Pump Lasers and THz Frequency Monitoring}

Aside from the photomixing diode, the most important components in the $\mathrm{THz}$ sweep oscillator are the two laser sources. They must provide single-frequency operation in a useful spatial mode, and one of them must be tunable by at least $1 \mathrm{THz}$. Fortunately, during the past decade great advances have occurred in single-frequency widely tunable lasers made from solid-state and semiconductor materials. Solid-state materials such as Ti: $\mathrm{Al}_{2} \mathrm{O}_{3}$ can easily be made to operate at a single frequency and have very high gainbandwidth product, so can provide high levels of power tunable over $\sim 10 \mathrm{~nm}$. Semiconductor diode lasers are more challenging but have been successfully developed with single-frequency operation using various optical techniques such as distributed Bragg reflectors and external gratings.

The lasers used in the THz sweep oscillator are the GaAs/AlGaAs-based masteroscillator power amplifiers (MOPAs) shown in the block diagram of the sweep-oscillator spectrometer in Fig. 1. They were developed by SDL Inc. (Model TC40) during the 1990s partly for the $820-900$ nm "first" window of fiber-optic telecommunications. ${ }^{35}$ The MOPA cleverly separates the functions of frequency generation and amplification, similar to what is done in microwave traveling-wave-tube amplifiers. This allows singlefrequency performance to be achieved with impressively high cw output power up to 
$\sim 0.5 \mathrm{~W}$. And the TC40 produces a nearly-Gaussian TEM spatial mode having a spot diameter of $\omega=1.8 \pm 0.3 \mathrm{~mm}$ (at the $1 / \mathrm{e}^{2}$ points), a beam divergence $<1 \mathrm{mrad}$ and a horizontal purely-linear polarization.

In the THz sweep oscillator only one of the two required MOPAs needs to be tunable. Continuous tuning between $\sim 779$ and $783 \mathrm{~nm}$ is achieved by a combination of course and fine electromechanical control of the master-oscillator cavity length. The course control is provided by a stepper motor with linear variable differential transformer (LVDT), which creates smooth and accurate translation over mm of range. The fine control is provided by a piezoelectric transducer stack, which accurately translates a few microns corresponding to a fine-tuning range of $\approx 90 \mathrm{GHz}$.

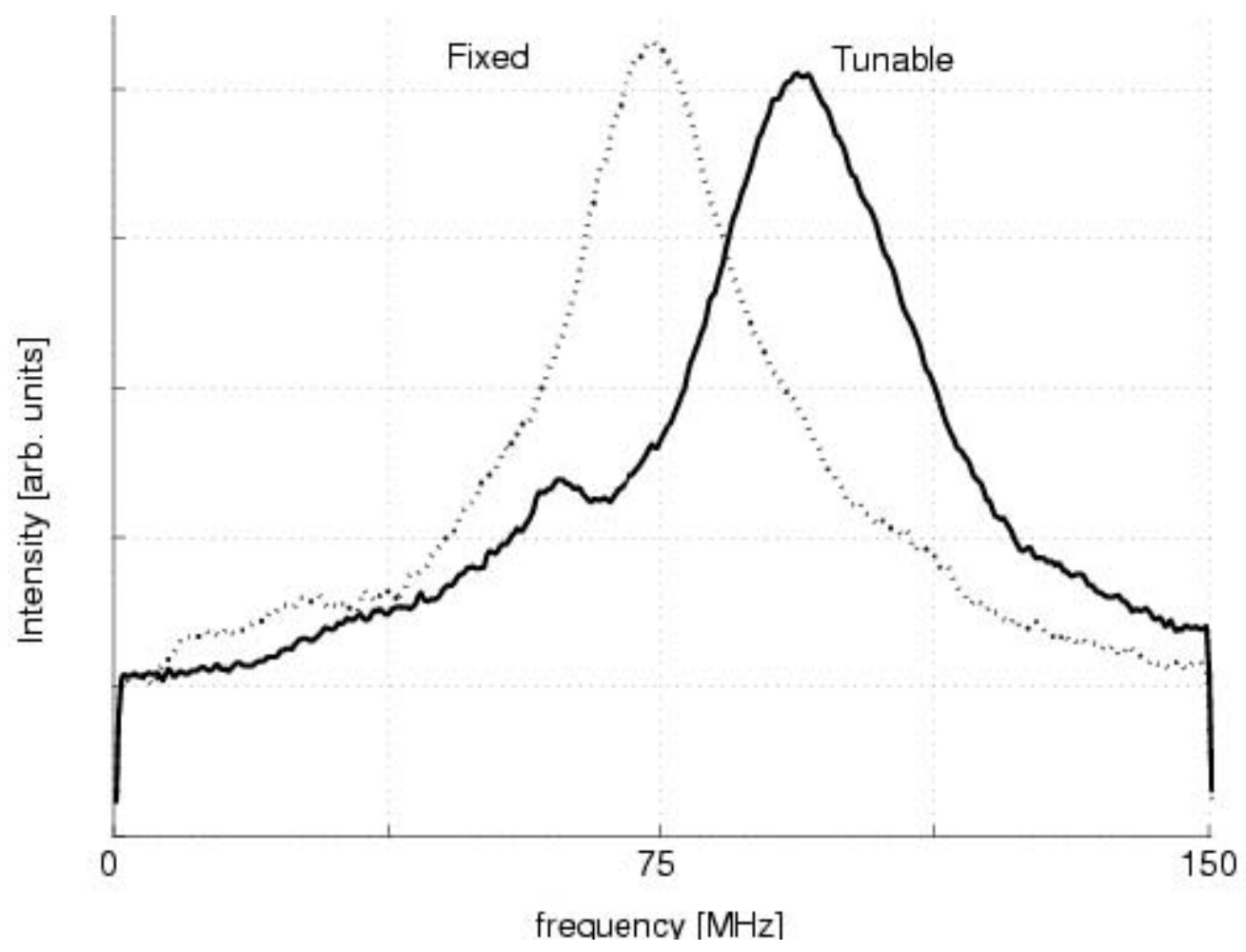

Fig. 20 Optical power spectrum of tunable and fixed-frequency MOPA lasers taken with confocal Fabry-Perot spectrometer.

The second MOPA is kept fixed in wavelength at a point of high stability, which is 
found to be $774.68 \mathrm{~nm}$. Both lasers are temperature-stabilized using thermoelectric coolers but are otherwise free-running and have no optical or electrical feedback.

To accurately resolve narrow spectral features such as the molecular absorption lines of gases in the atmosphere, it is necessary to know the laser difference frequency $\Delta v$ to better than $1 \mathrm{GHz}$. This is a task that becomes increasingly laborious and expensive as the stability of the lasers improves. So a simplifying design decision was to monitor the frequency only to the resolution $\delta v$ of a low-cost Fabry-Perot (FP) spectrum analyzer. . The FP analyzer contains a high-Q, 1-cm-long confocal cavity having a free spectral range (FSR) of $7.5 \mathrm{GHz}$ and an instrumental resolution of $37 \mathrm{MHz}$.

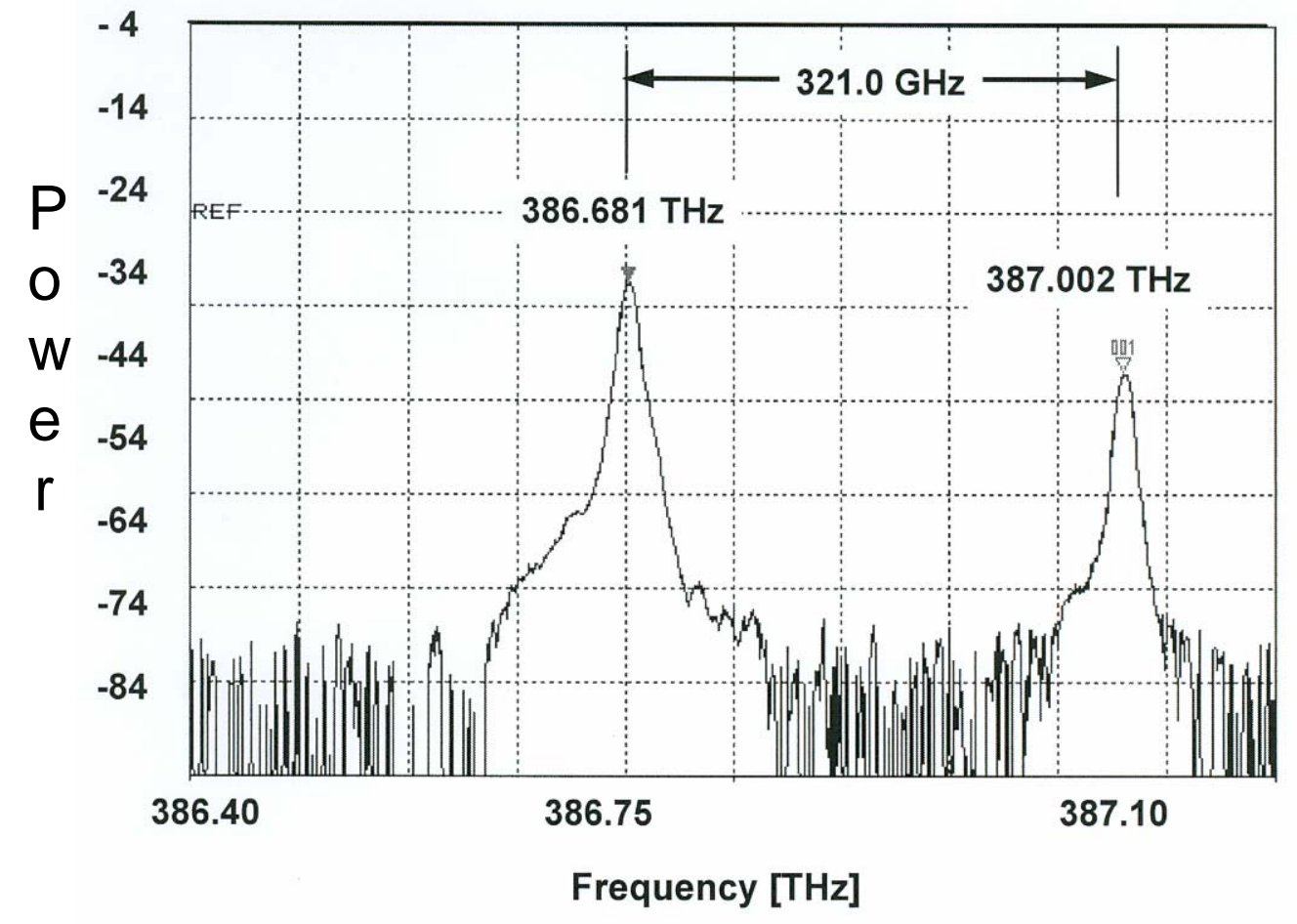

Fig. 21 Power spectrum of tunable and fixed-frequency MOPA lasers taken with fiberoptically-coupled optical spectrum analyzer.

The measured spectrum of the fixed and tunable MOPA lasers is plotted in Fig 20, showing a full-width at half-maximum (FWHM) of $35 \mathrm{MHz}$. This is remarkably 
comparable to the instrumental resolution, and it represents an upper limit on the linewidth of the MOPA lasers.

Given the 35-MHz upper limit for the FWHM of the free-running MOPA lasers, an effective frequency-monitoring technique is the dual spectrometer method in

Fig. 21. A commercial optical spectrum analyzer (OSA) designed for the range 650 to $1750 \mathrm{~nm}$ constantly measures the emission from the two MOPAs through the monitor port of Fig. 21. Because it is based on a grating spectrometer, the OSA has a modest resolution of $2.0 \mathrm{GHz}$. But it monitors both lasers simultaneously during a 100-nmwide sweep, and computes the difference with an internal microprocessor. The difference frequency is reported within 500 ms over the IEEE-488 bus to the master computer. To improve the accuracy further, a fraction of the laser power in the monitor port is coupled through the FP laser analyzer.

\subsection{Control and Signal Processing}

The THz sweep oscillator is controlled through a laptop computer running Matlab Version 6.0 under Windows 2000. The tunable MOPA, OSA, lock-in amplifier, and Fabry-Perot digitizer are all accessed through the GPIB (IEEE-488) bus using the gpib.m Matlab function. For example, to sweep the frequency of a photomixing $\mathrm{THz}$ source from $100 \mathrm{GHz}$ to $1500 \mathrm{GHz}$, the fine control (piezoelectric-stack voltage) of the tunable MOPA is set to zero and the course control (LVDT) is set so that the difference wavelength between the two lasers is about $0.2 \mathrm{~nm}$, corresponding to a photomixing frequency of $\sim 100 \mathrm{GHz}$. The fine control is then varied from 0 to $100 \mathrm{~V}$ corresponding to a 100 -to-175 GHz sweep at the difference frequency. When this sweep is completed, 
the LVDT is changed by $0.1 \mathrm{~nm}$ and another scan is made from 150 to $225 \mathrm{GHz}$. This procedure is iterated until the desired THz range is covered.

The fine control has two important parameters: step voltage and dwell time at each step. The step voltage is chosen to match the desired resolution of the scan consistent with the (jitter-limited) stability of the MOPAs of $\sim 100 \mathrm{MHz}$. For example, to achieve $1 \mathrm{GHz}$ steps, the fine control is varied between 0 and $100 \mathrm{~V}$ in 1.33-V steps. The dwell time is dictated mostly by the required integration time of the lock-in amplifier, which is typically 0.3 second between $100 \mathrm{GHz}$ and $1.0 \mathrm{THz}$, and $1.0 \mathrm{~s}$ above $1.0 \mathrm{THz}$ (because of diminishing signal strength). The dwell time is set equal to the integration time, creating a small lag between the signal acquired and its actual frequency. This lag poses a problem only for high-resolution scans and is usually mitigated in such cases by repeated scanning with longer dwell time or "smart scanning” as discussed further below.

One drawback to using the OSA is the time delay incurred by its sweeping the infrared spectrum and using a peak search algorithm to determine the wavelength (accurate to $2 \mathrm{GHz}$ ). This process simply takes too much time if done at every point of the sweep. So a compromise is made by acquiring the wavelength from the OSA at the two extreme voltages of each fine scan and interpolating linearly to find the wavelength in-between. Each data acquisition and computation from the OSA takes about $15 \mathrm{~s}$. When added to the dwell time of all the data points, a typical fine scan of $75 \mathrm{GHz}$ in 100 steps takes about 2 minutes total. Hence, a 100-to-1000 THz scan is completed in less than 40 min. 


\section{9. “Smart” Frequency Scanning}

One of the realities of $\mathrm{THz}$ photoconductive mixing is the inevitable drop in power with frequency. There is an associated drop in signal-to-noise ratio since the noise floor of either the HEB or Golay detectors remains practically constant with frequency. To counteract this effect, the integration time on the lock-in amplifier can be varied dynamically to keep the post-detection signal-to-noise ratio approximately constant. From fundamental signal-processing theory, we know that the signal-to-noise ratio after

lock-in detection is greater than that before detection by the factor $(B \tau)^{1 / 2}$ where $\mathrm{B}$ is the input bandwidth and $\tau$ is the lock-in sampling time. So as the sweep oscillator $\mathrm{THz}$ power drops, the SNR after lock-in amplification can be maintained approximately constant by adjustment of $\tau$.

A good example of the CSNR mode is shown in Fig. 22 which shows the background signal from the Golay cell between $100 \mathrm{GHz}$ and $1.5 \mathrm{THz}$. Between 100 $\mathrm{GHz}$ and $1.0 \mathrm{THz}$, the sampling time was $300 \mathrm{~ms}$, and between 1.0 and $1.5 \mathrm{THz}$ it was increased to $1 \mathrm{~s}$. The resulting spectrum appears "equally noisy” at all points on the spectrum. The penalty, of course, is the inability of the receiver to respond to rapid changes in the transmit power if they happen to occur.

Rapid changes in transmit power are expected for sharp spectral signatures, such as the resonant absorption lines of gases. The "dwell time” on any signature is approximately given by $\Delta v / \mathrm{R}$, where $\Delta v$ is the width [GHz] and R is the scan rate [GHz/s]. For example, in the background scan of Fig. 22, the scan rate is $70 \mathrm{GHz}$ per $40 \mathrm{~s}$. The number of samples taken by the receiver during the dwell time is simply 
$\mathrm{R} \cdot \tau / \Delta v$. It is important that this number remain consistent with the Nyquist sampling theorem, i.e., at least two samples are taken per cycle of the highest Fourier component in the power spectrum of the signature. Since $\tau$ is being varied to maintain

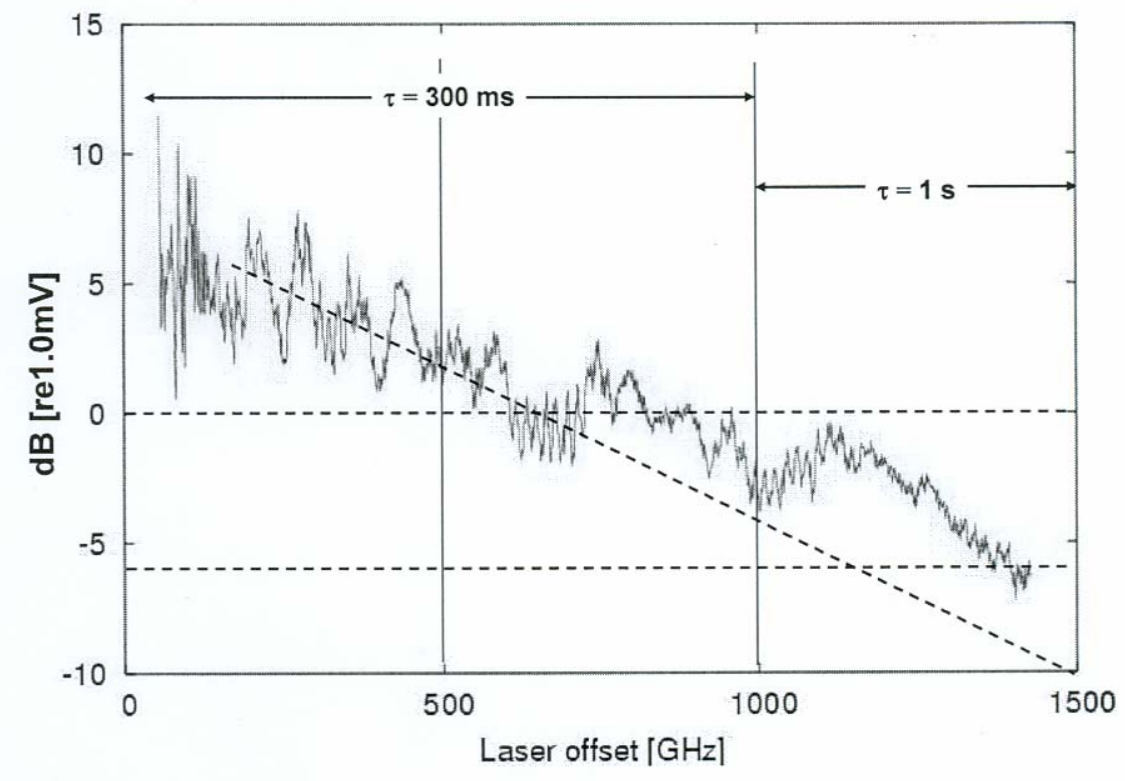

Fig. 22 Photomixer output power vs offset frequency as measured by the Golay cell and recorded by the lock-in amplifier. The scan from $100 \mathrm{GHz}$ to $1 \mathrm{THz}$ is made with an integration time $\tau$ of $300 \mathrm{~ms}$, and the scan above $1 \mathrm{THz}$ is made with a $\tau$ of $1 \mathrm{~s}$, yielding an approximately constant signal-to-noise ratio (CSNR) across the entire scan.

Approximately constant SNR, R must also be varied to satisfy Nyquist's criterion.

This is most easily done on the high-SNR HEB receiver that has lower standing-wave ratio and fewer sharp features to cause unnecessary reduction in $\mathrm{R}$. 


\section{Examples of $\mathrm{THz}$ Spectroscopy}

The first application of the photomixing sweep oscillator has been $\mathrm{THz}$ spectroscopy of materials under "STP” (standard temperature and pressure) conditions. Unlike the common techniques of Fourier transform and time-domain spectroscopy, sweep oscillator spectroscopy can selectively interrogate regions of the spectrum where interesting absorption features occur and ignore other regions. For many solids and liquids, the THz absorption is relatively weak but broad and monotonic. This class of materials tends to be the nonpolar dielectrics such as plastics, ceramics, and nonpolar semiconductors. For these materials, the photomixer can usually be operated in broadband mode using a Golay-cell detector and fast scanning. For polar gases and certain solids, the THz absorption can display resonant peaks of $\sim 1 \mathrm{GHz}$ width and large depth. Here, the sweep oscillator is best operated in slow-scan, narrowband mode using the HEB receiver and "smart" scanning.

The materials are all mounted in a stainless steel cylindrical sample holder that is 0.25-inch thick, 1.0 -cm in diameter and covered with 0.0005-inch-thick polyethylene windows. Polyethylene is one of the most transparent of all solid materials in the $\mathrm{THz}$ region and introduces negligible standing waves over the frequency range (100 GHz to 2 $\mathrm{THz}$ ) of the present experiments. All materials are tested in packed-powder form to minimize scattering and maximize homogeneity within the sample holder.

\subsection{Fast-scan, broadband mode}

Fig. 23 shows the normalized transmission spectra obtained using the sweep oscillator in fast-scan, broadband mode on three types of materials of interest to 
biodetection: (1) simple organic monomolecular materials and polymers, (2) macromolecular materials, and (3) bioparticles. The simple organic materials include sodium bicarbonate (baking soda and Teflon). The macromolecular include two materials rich in polysaccharides and proteins: the most common "building blocks" of the biological world. They are corn starch and wheat flour. Wheat flour is composed of $\sim 40 \%$ starch, the remainder being protein and inorganic material. The bioparticles include spores of Bacillus subtilus (BG) (an Anthrax surrogate) and bakers yeast (Saccharomyces cerevisiae). The yeast is tested in two forms: (1) in 1-mm-diam pellets as delivered commercially, and (2) ground up into a powder having the same consistency and roughly the same density as the BG.

The trend in Fig. 23 is clear and consistent with the dozens of organic materials measured by the sweep oscillator to date. The monomolecular organics and inorganics have the weakest absorption at THz frequencies, and the starch- and protein-laden materials have the strongest. It is somewhat surprising just how big the difference is. To quantify the absorption, Fig. 24 shows the transmission for potato starch on a semilogarithmic plot. 


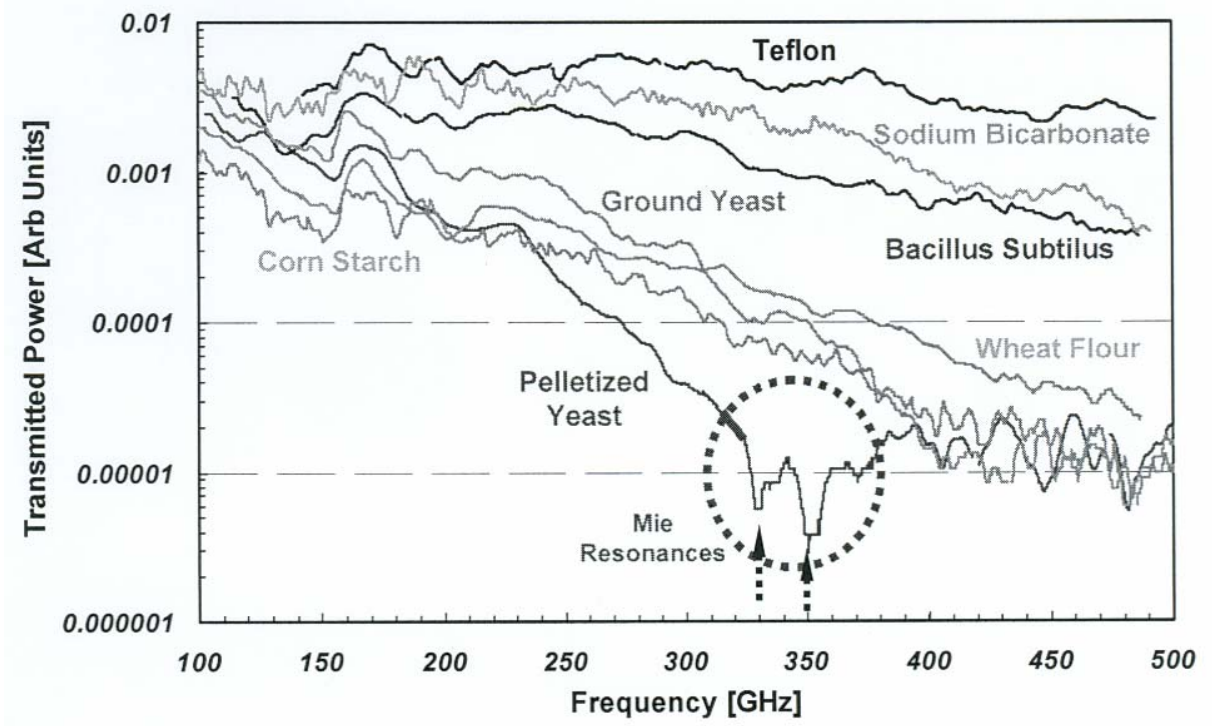

Fig. 23 Example of the use of photomixer spectroscopy on common organic and biomaterials. All scans were made in fast-scan broadband (i.e., low resolution) mode.

If we assume the applicability of the Lambert-Beer law, $\mathrm{T}=\mathrm{e}^{-\alpha \mathrm{z}}$ where $\alpha$ is the absorption coefficient, then this plot represents $\alpha$ vs. f. The transmission curve displays a slope of $\approx 2.0$, meaning $\alpha \propto \mathrm{f}^{2}$. This is a trend we (and others) have seen often in the absorption of solids in the THz region. ${ }^{36}$ A quadratic dependence can be explained from classical electromagnetics and the Lorentzian model of dipole transitions, assuming that the frequency of measurement lies well below an arbitrary number of Lorentzian absorption resonance. 


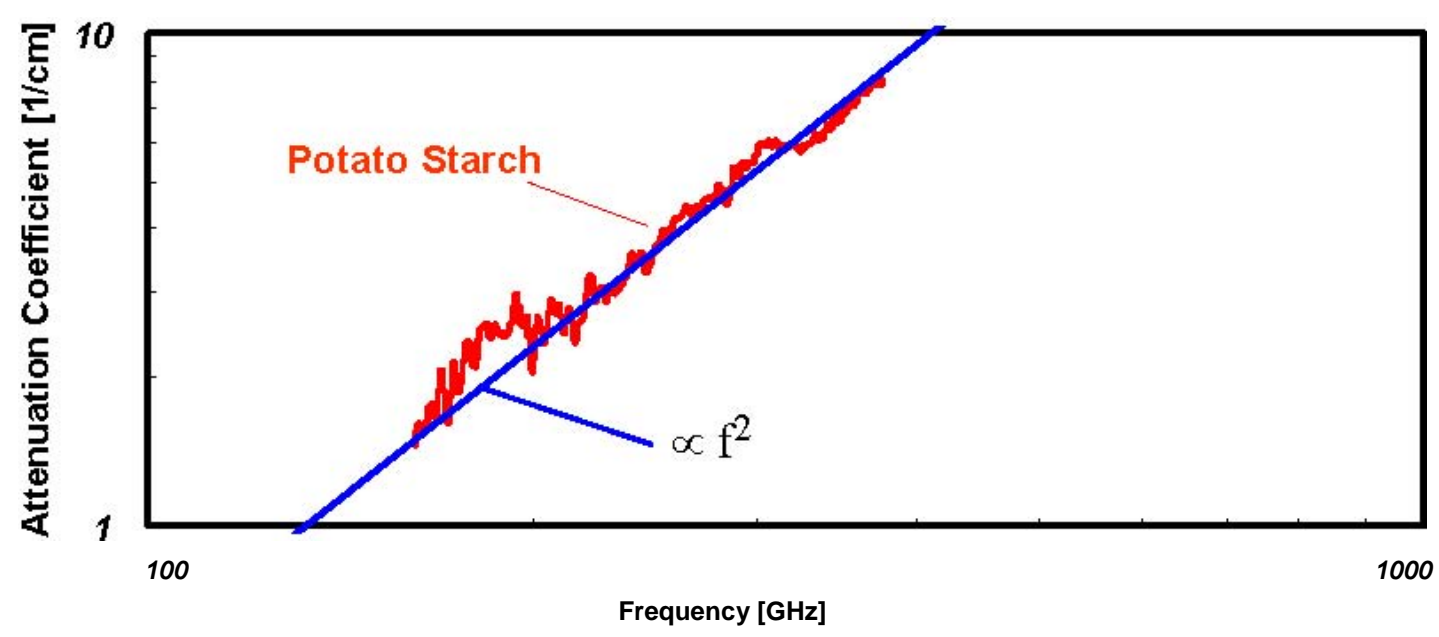

Fig. 24 The attenuation coefficient of potato starch vs frequency derived from its fast-scan broadband transmission spectrum. Also shown is a quadratic frequency dependence fit.

The bioparticles show an intermediate attenuation with the Bacillus subtilus being significantly more transparent than both forms of yeast. For example, the powdered yeast sample shows at least 10 -dB more attenuation than the Teflon starting at $\approx 300 \mathrm{GHz}$, and at least $10 \mathrm{~dB}$ more attenuation than the BG sample starting at $\approx 400 \mathrm{GHz}$. The palletized yeast sample displays an even stronger attenuation, largely because of a broad absorption feature between roughly 250 and $400 \mathrm{GHz}$. Examination of Fig. 23 indicates two resonant peaks within this feature centered around 330 and $350 \mathrm{GHz}$, respectively. These may be associated with Mie resonances in the pellets, which are approximately prolate spheroids having a semi-major axis of 0.4-to-0.5 mm and a semi-minor axis of 0.3-to-0.4 $\mathrm{mm}$. Electromagnetic scattering theory predicts three distinct resonances for ellipsoidal particles in general, which reduces to two resonances for the special case of a spheroid. 


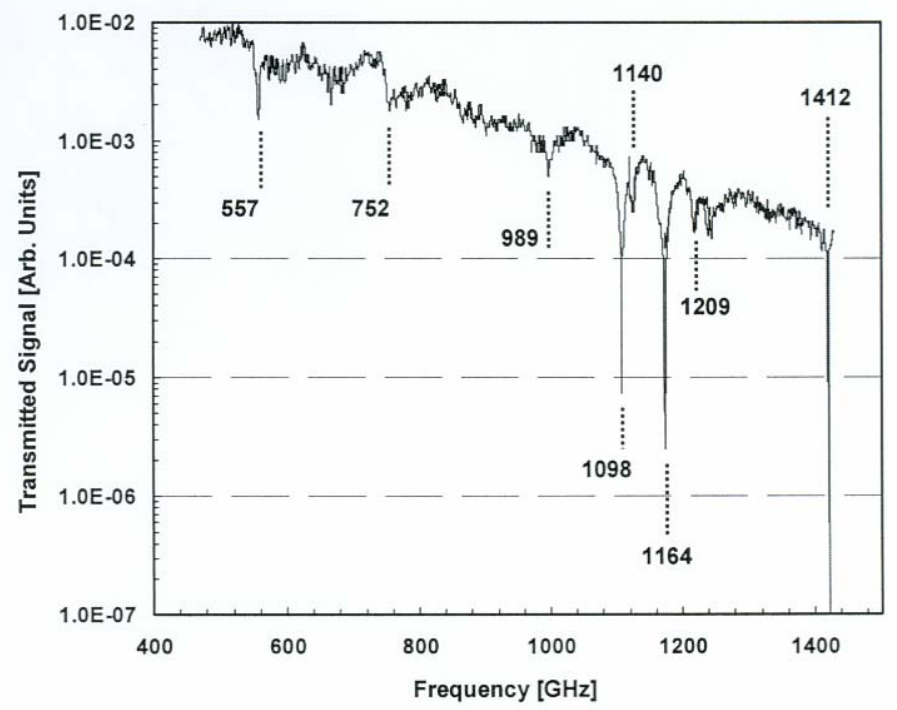

Fig. 25 Example of a 1-THz scan taken with photomixing spectrometer in slow-scan, narrow-band mode. The medium is the atmosphere in our laboratory over a path length of $15.5 \mathrm{~cm}$.

\subsection{Slow-scan, narrow band mode}

Shown in Fig. 25 is the atmospheric spectrum taken through the brass sample tube of path length $0.155 \mathrm{~m}$ at $65 \%$ relative humidity and $72^{\circ} \mathrm{C}$. The scan extends between 400 and $1450 \mathrm{GHz}$. It displays several discrete absorption lines, the strongest attributable to water vapor. The strongest three lines by far are those centered near 1098, 1164, and $1412 \mathrm{GHz}$. They are representative of a set of strong water lines in the THz region and are the primary factor behind the THz region being relatively opaque compared to the microwave and millimeter-wave bands below. Table II lists the center frequencies and line-center absorption coefficients for each of these lines computed from the kinetic coefficients and a radiative transfer algorithm. Note that over a path length of just $1 \mathrm{~m}$, the lowest-frequency line listed absorbs over $18 \mathrm{~dB}$ at line center. 
Table 2. Ten strongest water lines below 2

\begin{tabular}{|l|l|}
\hline Frequency [] & Absorption Coefficient [dB/km] \\
\hline 0.5576 & 18354 \\
\hline 0.7528 & 12677 \\
\hline 0.9888 & 9370 \\
\hline 1.0982 & 61582 \\
\hline 1.1637 & 71511 \\
\hline 1.4117 & 52841 \\
\hline 1.6712 & 163625 \\
\hline 1.7183 & 150085 \\
\hline 1.8690 & 59902 \\
\hline 1.9205 & 46351 \\
\hline
\end{tabular}

Fig. 26 displays the same atmospheric transmission spectrum but with "zoom-in” scans taken around two of the strongest water lines at 557 and $1164 \mathrm{GHz}$. The zoom-in shows clearly the effect of pressure broadening to a full-width at half-maximum of approximately $3 \mathrm{GHz}$ for both lines. But neither line shape is exactly the Gaussian expected from pressure-broadening theory. This is probably because the lines are both optically thick, and there are other transitions taking place in the wings, such as the shoulder on the low-frequency side of the 1164-GHz line. In any case, this plot accentuates the advantages of the sweep-oscillator instrument compared to all other known THz spectrometers. 


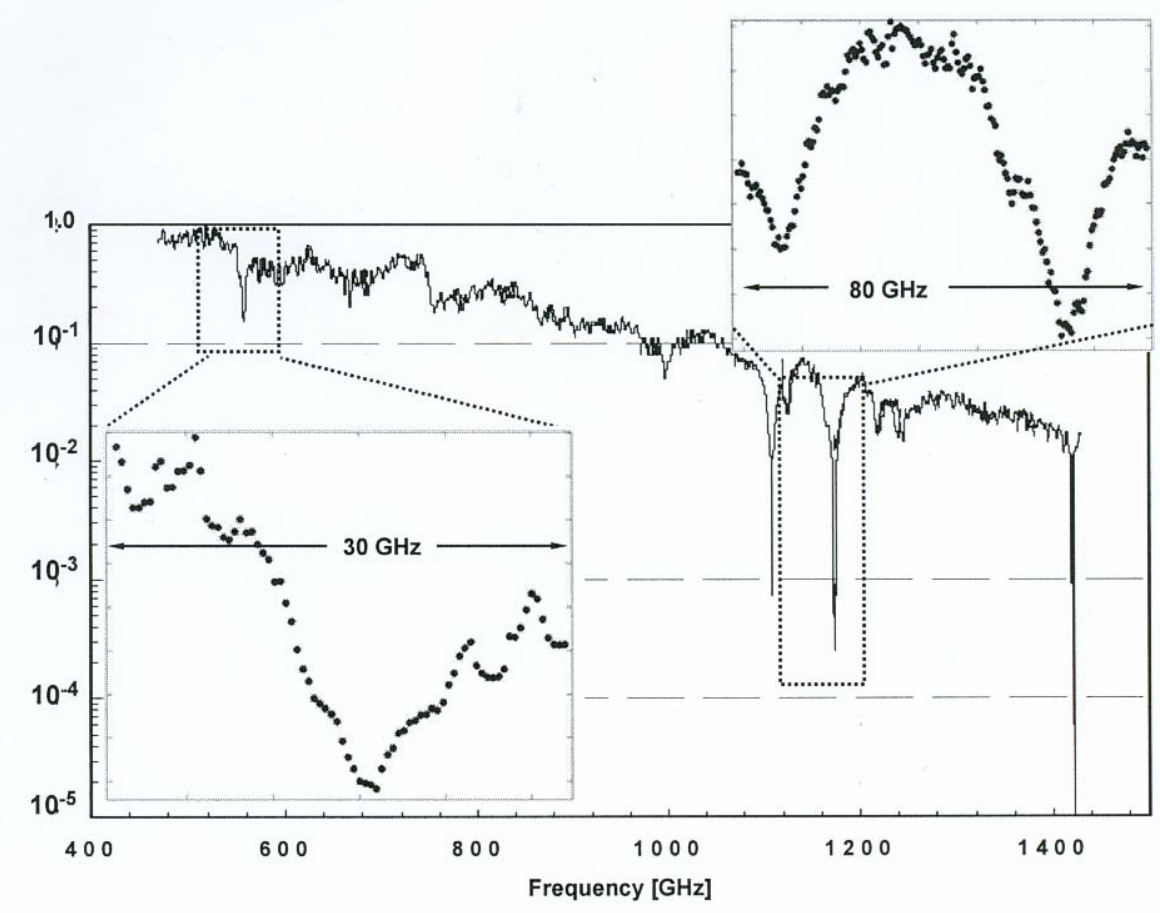

Fig.26 The same scan as Fig. 24 with zoom-in on two of the strong water-vapor absorption signatures.

\section{Technology Transfer}

Our group brings considerable capabilities and expertise in $\mathrm{THz}$ devices, components, and measurements in support of the Goodrich Optical Systems and Space Division (Danbury, CT). Our goal is to characterize biomaterials at THz Frequencies for Homeland Security. The research objective is to obtain signature data in the $60 \mathrm{GHz}$ to $300 \mathrm{GHz}$ regions of the spectrum for several biological agent simulants and interferants. Signature data is desired for the following simulants. Simulants are listed in order of priority.

\footnotetext{
- Toxin - Ovalbumin

- $\quad$ Virus - MS2

- Vegetative Bacteria - Erwinia herbicola

. $\quad$ Bacterial spore - Bacillus globigii
} 


\section{Scientific personnel" supported by this project and}

\section{honors/awards/degrees received}

(a) Mihail Sukhotin, completed M.S. degree Electrical Engineering at UCLA in 2003

(b) Sanjeev Murthy, completed Ph.D. degree Electrical Engineering at UCLA in 2003

(c) Jon E. Bjarnason, completed M.S. degree Electrical Engineering at UCLA in 2004

(d) Tak Ling Chan, Staff Engineer

(e) Marie A. Celis, Staff Research Associate

(f) Elliott R. Brown, Principal Investigator

(g) Ming Wu, Co-Principal Investigator

\section{Acknowledgements}

This work was sponsored by the U.S. Air Force (Mr. Jim Nichter) through the DARPA THz Program.

\section{References}

1. A. Tredicucci, F. Capasso, C. Gmachi, D.L. Sivco, A.L. Hutchison, and A.Y. Cho, Appl. Phys. Lett., 73 (15), pp. 2101-2103 (1998).

2. M. Rochat, J. Faist, M. Beck, U. Oesterle, and M. IllegemsAppl. Phys. Lett, Vol. 73, p. 3724 (1998).

3. G. Scalari, L. Ajili, J. Faist, H.E. Beere, E.H. Linfield, D.A. Ritchie, and A.G. Davies, Appl. Phys. Lett. 82, 3165 (2003).

4. B. S. Williams, H. Callebaut, S. Kumar, Q. Hu, and J. L. Reno, Appl. Phys. Lett. Vol. 82, p. 1015 (2003).

5. R. L. Aggarwal, B. Lax, H. R. Fetterman, P. E. Tannenwald, and B. J. Clifton, Appl. Phys. Lett, vol. 45, p. 3972 (1974).

6. D.W. Porterfield, T.W. Crowe, R.F. Bradley, N.R. Erickson, Proc. 1998 IEEE MTT Intermational Microwave Symposium.

7. J. Bruston, R.P. Smith, S.C. Martin, and P.H. Siegel, Proc. 1998 IEEE MTT Intermational Microwave Symposium.

8. I. Mehdi, E. Schlecht, G. Chattopadhyay and Peter H. SiegelFar-IR, Sub-MM \& MM 
Detector Tech. Workshop, Monterey, CA, 3/2002.

9. Y. J. Ding and J. B. Khurgin, Opt. Commun., 148, 105 (1998).

10. F.W. Smith, Ph.D. Thesis, Massachusetts Institute of Technology, 1990.

11. C. Kadow, S.B. Fleischer, J.P. Ibbetson, J.E. Bowers, A.C. Gossard, J.W. Dong, and C.J. Palmstrom, Appl. Phys. Lett., vol. 75, p. 3548 (1999).

12. E.R. Brown, Int. Journal of High Speed Electronics and Systems, Vol. 13 (World Scientific, 2002).

13. A. Jackson, "Low-Temperature-Grown GaAs Photomixer Designed for Increased THz Output Power,” Ph.D. Thesis, University of California, Santa Barbara, 1999.

14. provided by Dr. H. Kazemi and Rockwell Scientific, Thousand Oaks, CA

15. provided by Dr. P.H. Siegel, Jet Propulsion Laboratory.

16. E. R. Brown, "THz Generation by Photomixing in Ultrafast Photoconductors", International Journal of High Speed Electronics and Systems, Vol. 13, pp. 497-545 (2003).

17. S. M. Duffy, S. Verghese, K. A. McIntosh, A. Jackson, A.C. Gossard, and S. Matsuura, IEEE Trans. Microwave Theory and Tech., vol. 49, p. 1032 (2001).

18. D.C. Driscol, M. Hanson, C. Kadow, and A.C. Gossard

19. E. R. Brown, K. A. McIntosh, F. W. Smith, M. J. Manfra, C. L. Dennis. Measurement of optical-heterodyne conversion in low-temperature-grown GaAs. Appl. Phys. Lett. 62(11):1206-8, 1993.

20. A.W. Jackson, Ph.D. Thesis: Low-Temperature-Grown GaAs Photomixers for Increased Terahertz Output Power, October 1999.

21. E.R. Brown,, " Generation by Photomixing in Ultrafast Photoconductors,” Int. Journal of High Speed Electronics and Systems, Vol. 13 (World Scientific, 2002).

22. A. S. Pine, R.D. Suenram, E. R. Brown, and K. A. McIntosh, J. Mol. Spect.,vol. 175, p. 37 (1995).

23. K. A. McIntosh and S. Verghese, Proceedings of SPIE, vol. 3794 , pp. 16-24 (1999).

24. E.R. Brown, Appl. Phys. Lett., vol. 75, p. 769 (1999).

25. A.W. Jackson, Ph.D. Thesis, University of California, Santa Barbara, 1999. 
26. J. Bjarnason, et al., submitted to Appl. Phys. Lett., October 2003.

27. E.R. Brown, K.A. McIntosh, K.B. Nichols, and C.L. Dennis, Appl. Phys. Lett., vol. 66, pp. 285-287 (1995).

28. S.M. Duffy, S. Verghese, K.A. McIntosh, A. Jackson, A.C. Gossard, and S. Matsuura, IEEE Trans. Microwave Theory and Tech, vol. 49, pp. 1032-1038 (2001).

29. A.W.M. Lee et al. 2003, in preparation.

30. D.B. Rutledge, D.P. Neikirk, and D.P. Kasilingam, in Infrared and Millimeter Waves (Academic, Orlando, FL, 1983), Vol. 10, p. 1.

31. D. F. Filipovic, G.P. Gauthier, S. Raman, and G.M. RebeizIEEE Trans. On Antennas andPropagation, vol. 45, pp. 760-766 (1997).

32. A. Yariv, (Wiley, New York, 1975).

33. see, www.josephson.terahertz.co.uk/QMCI/GOLAY.HTM

34. courtesy of Dr. Peter Siegel, Jet Propulsion Laboratory.

35. S. V. Kartalopoulos, (IEEE Press, New York, 2000), Sec. 3.19.

36. T. L. J. Chan, J. E. Bjarnason, A. W. M. Lee, M. A. Celis, and E. R. Brown, Applied Physics Letters, in press.

37. C.F. Bohren and D.R. Huffman (Wiley, New York, 1983).

38. Obtained with PCLnWin from Ontar Corp., and the HITRAN96 database. 